\title{
2-胺基苯并噁唑衍生物合成的研究进展
}

\author{
游利琴袁金伟*杨亮茹肖咏梅毛璞 \\ (河南工业大学化学化工学院 河南省天然药物化学院士工作站 郑州 450001)
}

\begin{abstract}
摘要 2-胺基苯并噁唑衍生物是一类重要的杂环化合物, 在医药、生物和材料科学方面具有较好的用途, 因此其合成方 法备受关注. 近年来，文献报道了在过渡金属或非金属催化下等一系列高效、绿色的合成 2-胺基苯并噁唑衍生物方法. 按照不同的反应原料和合成方法进行分类，综述了近年来 2-胺基苯并噁唑衍生物合成研究的新进展.

关键词 2-胺基苯并噁唑; 过渡金属催化; 非金属催化; 合成; 研究进展
\end{abstract}

\section{Progress in the Synthesis of 2-Aminobenzoxazole Derivatives}

\author{
You, Liqin $\quad$ Yuan, Jinwei* $\quad$ Yang, Liangru $\quad$ Xiao, Yongmei $\quad \mathrm{Mao}, \mathrm{Pu}$ \\ (Academician Workstation for Natural Medicinal Chemistry of Henan Province, School of Chemistry \& Chemical \\ Engineering, Henan University of Technology, Zhengzhou 450001)
}

\begin{abstract}
Aminobenzoxazole derivatives are a kind of heterocyclic compounds, which play important roles in medicine, biology and material science, and their synthetic methods have attracted much attention. In recent years, many efficient, green synthetic approaches of 2-aminobenzoxazole derivatives using transition-metal or metal-free catalytic systems have been reported. Based on differences of reaction substrates and synthetic methods, the recent advances in the synthesis of 2-aminobenzoxazole derivatives are reviewed.
\end{abstract}

Keywords 2-aminobenzoxazole; transition-metal catalyzed; metal-free catalyzed; synthesis; research progress

苯并噁唑是一种含有 $\mathrm{N} 、 \mathrm{O}$ 杂原子的苯并杂环化合 物, 具有抗菌、抗炎、抗肿瘤等生物活性, 而且具有特 殊的光学性能, 这类化合物在医药、农药、生物及材料 等领域具有广泛的用途, 是一种可以用来开发多种新药 的前体化合物 ${ }^{[1 \sim 3]}$. 苯并噁唑骨架连接的取代基和位置 对其性质有较大的影响, 构效关系研究表明 2-位上取代 基的类型对其生理活性的影响较大 ${ }^{[4]}$. 因此, 近些年来, 人们对苯并噁唑的 $C(2)$ 位进行了多种功能化修饰, 包括

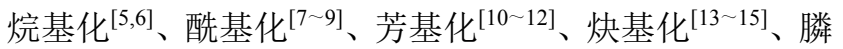
酰化 ${ }^{[16]}$ 和胺化 ${ }^{[17]}$ 等. 其中, 2-胺基苯并噁唑衍生物是一 类重要的具有较高生物活性的分子, 通过其衍生得到大 量的目标药物分子在抗艾滋病(AIDS)和肿瘤 ${ }^{[18]}$, 治疗 阿尔茨海默氏病、精神分裂症等神经性疾病 ${ }^{[19]}$ 具有较好 的效果; 此外, 2-胺基苯并噁唑衍生物在材料科学方面
也具有较好的用途 ${ }^{[20]}$. 因此, 2-胺基苯并噁唑衍生物的 合成方法受到人们的广泛关注.

迄今为止, 研究工作者们提出了合成 2-取代苯并琴 唑衍生物的多种方法. 2014 年，肖立伟等 ${ }^{[21]}$ 已对 2-取代 苯并噁坐衍生物的合成方法进行了详细报道, 然而对于 2-胺基苯并噁唑衍生物的合成方法综述，并未见到相关 文献报道. 本文针对近年来出现的 2-胺基苯并噁唑衍生 物的合成方法，依据反应原料、胺源和合成方法不同， 对近年来 2-胺基苯并㤠唑衍生物的合成进行了综述.

\section{1 以邻氨基苯酚衍生物为原料合成 2-胺基苯并 噁唑衍生物}

邻氨基苯酚衍生物是合成 2-胺基苯并噁唑衍生物 最常用的原料之一. 通常利用邻氨基苯酚衍生物与氧

\footnotetext{
*E-mail: yuanjinweigs@126.com

Received April 21, 2016; revised May 28, 2016; published online July 7, 2016.

Project supported by the National Natural Science Foundation of China (Nos. 21302042, 21172055), the Department of Henan Province Natural Science and Technology Foundation (No. 142102210410), the Natural Science Foundation in Henan Province Department of Education (No. 14B150053), the Program for Innovative Research Team from Zhengzhou (No. 131PCXTD605), and the Natural Science Foundation from Technology bureau of Zhengzhou City (No. 20130883).

国家自然科学基金(Nos. 21302042, 21172055)、河南省科技厅自然科学(No. 142102210410)、河南省教育厅自然科学(No. 14B150053)、郑州市科技创 新团队(No. 131PCXTD605)和郑州市科技局自然科学(No. 20130883)资助项目.
} 
类、胺类等化合物反应生成 2-胺基苯并噁唑衍生物. 例 如, 1998 年, Kaupp 等 ${ }^{[22]}$ 以邻氨基苯酚与 $\mathrm{BrCN}$ 为原料, 在无碱的条件下通过气一固相定量反应合成了 2-氨基苯 并噁唑溴酸盐; 然后加入碳酸钠中和 $\mathrm{HBr}$ 后生成目标产 物(Scheme 1). 反应可能是首先邻氨基苯酚与 $\mathrm{BrCN}$ 反 应生成邻羟基苯氰胺, 由于氰胺很容易受到亲核试剂的 进攻发生亲核加成, 因此差基通过分子内的亲核反应加 成到氰胺基官能团上生成 1 ; 然后加入碱溶液中和酸后 生成目标产物 2-胺基苯并噁唑(2). 该反应基本定量的 反应, 产率较高, 但是原料使用到剧毒性气体 $\mathrm{BrCN}$.

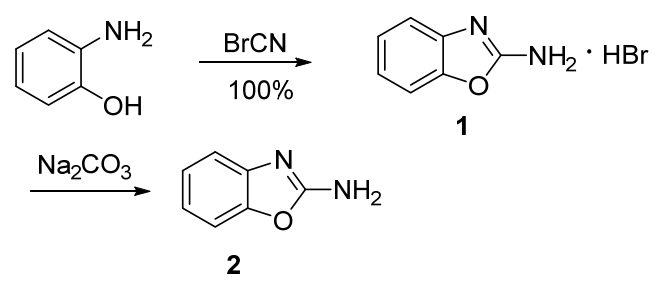

图式 1 化合物 2 的合成路线

Scheme 1 Synthetic route of compound 2

2003 年, $\mathrm{Wu}$ 等 ${ }^{[23]}$ 以取代邻氨基苯酚与二咪唑基甲 胺(DIMA)为原料, 在 THF 溶剂中回流 6 12 h 合成了 3 个 2-胺基苯并噁唑衍生物, 产率为 50\% 94\% (Scheme 2). 整个反应过程分为两个阶段进行: 首先取代邻氨基 苯酚与 DIMA 通过亲核取代反应脱去一分子咪唑, 生成 中间体 3; 然后 3 中的 $\mathrm{OH}$ 通过分子内的亲核取代反应, 再脱去一分子咪唑, 再经过分子内的重排生成目标化合 物 2 (Scheme 2). 该方法选用 DIMA 作为与取代邻氨基 苯酚反应形成目标化合物的碳源和氮源, 不仅能合成五 元杂环，而且能合成六元杂环化合物.

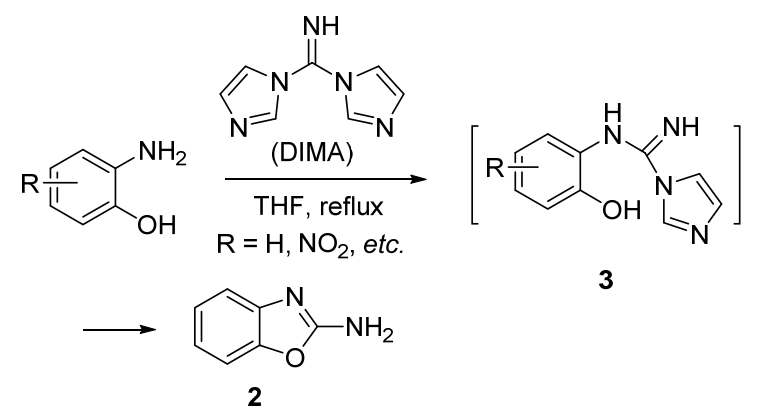

图式 2 化合物 2 的合成路线

Scheme 2 Synthetic route of compound 2

2006 年, EI-Faham 等 ${ }^{[24]}$ 以邻氨基苯酚与氯甲眯六 氟磷酸盐为原料, 三乙胺为缚酸剂, 在 $\mathrm{DMF}$ 溶剂中回 流 16 24 h 合成了 3 个 2-胺基苯并惡唑衍生物, 产率为 $59 \% \sim 70 \%$ (Eq. 1). 该反应可能的机理是：邻氨基苯酚 的氨基首先进攻氯甲眯六氟磷酸盐 4 与氯原子连接的碳 原子, 脱去 $\mathrm{HCl}$ 后形成过渡态 $\mathbf{5} ; \mathbf{5}$ 脱去 $\mathrm{HPF}_{6}$ 后生成过
渡态 6 , 由于二甲氨基是一个比较好的离去基团，因此 6 发生环合脱去二甲胺得到目标产物 2-胺基苯并噁唑衍 生物 7 (Scheme 3). 该方法操作简单、反应条件温和，但 是反应中使用的原料氯甲眯六氟磷酸盐不易得到，反应 时间较长，且有副反应发生.

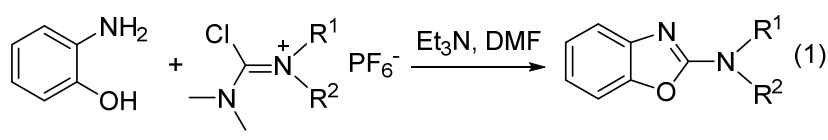

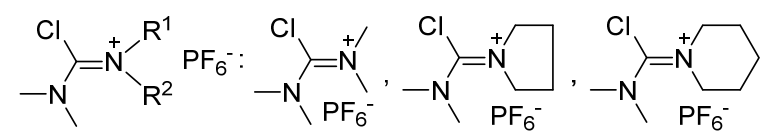

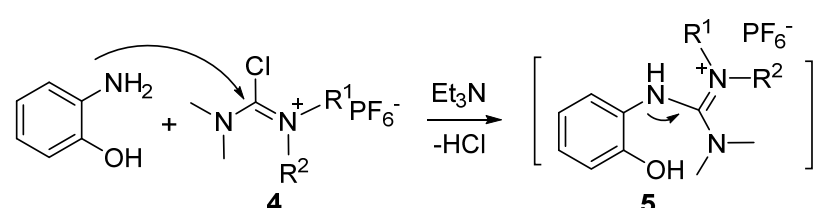

5

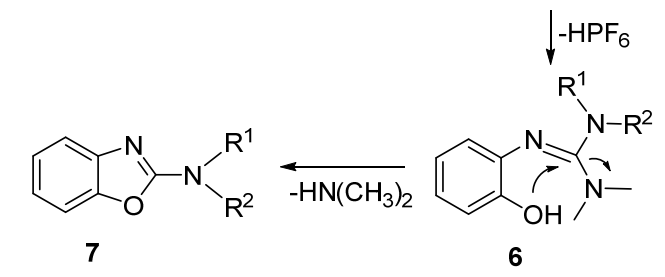

图式 3 合成 2-胺基苯并噁唑衍生物 7 的反应机理 Scheme 3 Reaction mechanism of synthesis of 2-aminobenzoxazole derivatives 7

2010 年, Cioffi 等 ${ }^{[25]}$ 以取代邻氨基苯酚与原碳酸四 甲酯、一级胺或二级胺、醋酸为原料, 在 $60{ }^{\circ} \mathrm{C}$ 的 $\mathrm{CHCl}_{3}$ 溶剂中反应 $16 \mathrm{~h}$; 或者以取代邻氨基苯酚与 1,1 -二氯二 苯氧基甲烷、一级胺或二级胺、三乙胺为原料，在甲苯 溶剂中室温下反应 $16 \mathrm{~h}$, 共合成了 22 个 2-胺基苯并惡 唑衍生物，产率为 $23 \% \sim 97 \%$ (Eq. 2). 该反应可能的机 理是：原碳酸四甲酯与邻氨基苯酚在醋酸作用下首先发 生缩合反应生成 8 ; 然后通过一个 $\mathrm{S}_{\mathrm{N}} \mathrm{Ar}$-类型的胺的取 代反应得到目标产物 7. 另一个可能的反应机理是：1,1二氯二苯氧基甲烷与邻氨基苯酚在三乙胺作用下发生 缩合反应生成 7; 然后通过一级胺或二级胺取代活性中 间体 9 中的苯氧基得到目标产物 7 (Scheme 4). 该反应 条件比较温和, 但是反应需要在酸性条件或碱性条件下 进行，底物的适用范围具有一定的局限性

2010 年, Carpenter 等 ${ }^{[26]}$ 以取代的邻氨基苯酚、苯基 异硫氰酸酯为原料, 在 $N$-环己基碳化二亚胺 $N^{\prime}$-甲基聚 苯乙烯作用下，在 DMF 溶剂中微波辐射下反应 $11 \mathrm{~min}$, 合成了 9 个 2-胺基苯并噁唑衍生物，产率为 $68 \%$ ～ $89 \%$ (Eq. 3); 2011 年, Zhang 课题组 ${ }^{[27,28]}$ 以取代的邻氨基苯 酚、苯基异硫氰酸酯为原料, 在 $\mathrm{FeCl}_{3} \cdot 6 \mathrm{H}_{2} \mathrm{O}$ 作用下, 以 


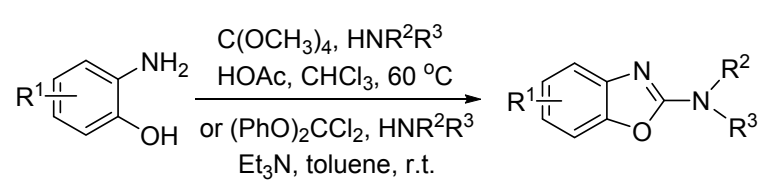

$\mathrm{R}^{1}=\mathrm{NO}_{2}, \mathrm{Cl}, \mathrm{OCH}_{3}, \mathrm{CH}_{3}, \mathrm{~F}$, etc.

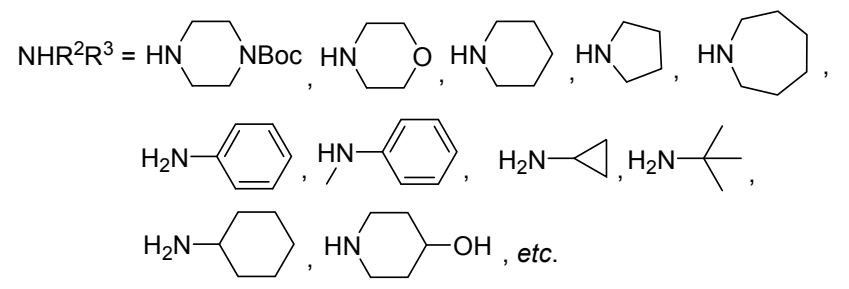

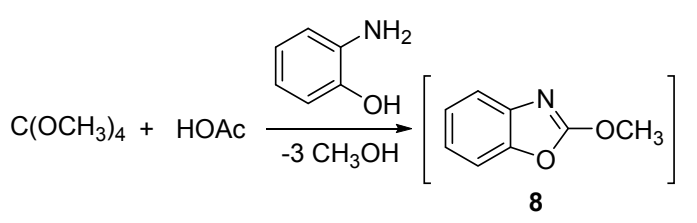

$\underset{-\mathrm{CH}_{3} \mathrm{OH}}{\stackrel{\mathrm{NHR}^{2} \mathrm{R}^{3}}{\longrightarrow}}$

7

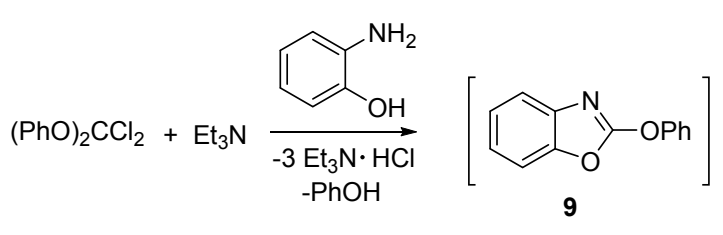

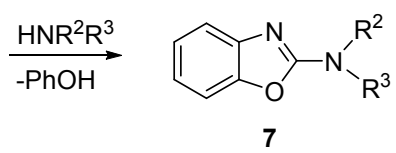

图式 4 合成 2-胺基苯并噁唑衍生物 7 的反应机理

Scheme 4 Reaction mechanism of synthesis of 2-aminobenzoxazole derivatives 7

$$
\begin{aligned}
& \mathrm{R}=\mathrm{CH}_{3}, \mathrm{Cl}, \mathrm{OCH}_{3}, \text { etc. } \\
& \mathrm{R}^{1}=\mathrm{C}_{6} \mathrm{H}_{5}, \mathrm{o}-\mathrm{CH}_{3}-\mathrm{C}_{6} \mathrm{H}_{4}, p-\mathrm{Cl}-\mathrm{C}_{6} \mathrm{H}_{4} \text {, etc. }
\end{aligned}
$$

水为溶剂在 $80{ }^{\circ} \mathrm{C}$ 反应, 合成了 15 个 2 -胺基苯并噁唑衍 生物, 产率为 $62 \% \sim 79 \%$ (Scheme 4). 该方法使用到有 毒的试剂苯基异硫氰酸酯为原料, 其适用范围受到一定 的限制.

2013 年, Singh 等 ${ }^{[29]}$ 以取代的邻氨基苯酚、脂肪族 的一级胺或二级胺和二硫化碳为原料, 在无催化剂和溶
剂的条件下，通过一锅者的方法在 $110{ }^{\circ} \mathrm{C}$ 反应 $3 \mathrm{~h}$, 合 成了 20 个 2-胺基苯并啞唑衍生物, 产率为 56\% 96\%. 该反应可能的机理是: 脂肪胺与 $\mathrm{CS}_{2}$ 发生加成后与第二 个脂肪胺分子形成中间体 10 ; 然后取代邻胺基苯酚与 (I)发生亲核加成得到中间体 11, 11 脱去一分子 $\mathrm{H}_{2} \mathrm{~S}$ 得到 中间体 $12 ; 12$ 发生分子内的亲核加成得到化合物 13 , 最 后失去一分子 $\mathrm{H}_{2} \mathrm{~S}$ 得到目标产物 14 (Scheme 5). 该合成 方法操作简便，条件较温和，但是该方法不适合以芳香 胺为原料合成 2-胺基苯并啞唑衍生物.

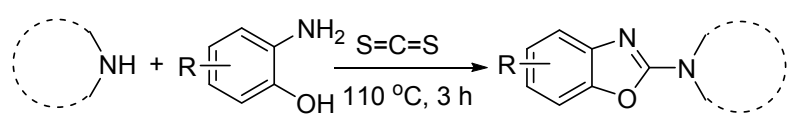

$\mathrm{R}=\mathrm{CH}_{3}, \mathrm{NO}_{2}$, et al.
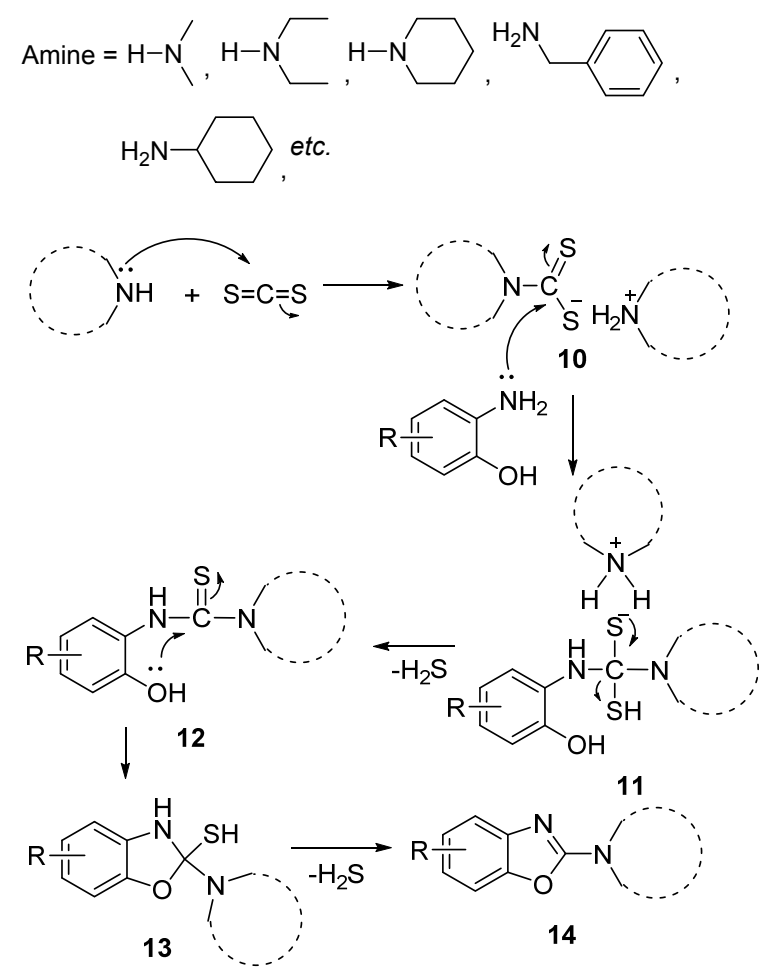

图式 5 化合物 14 的合成路线与反应机理

Scheme 5 Synthetic route and reaction mechanism for compound 14

2015 年, Kasthuri 等 ${ }^{[30]}$ 报道了取代的邻氨基苯酚与 亲电性的氰化试剂 $N$-氰基- $N$-苯基对甲苯磺酰胺(NCTS) 以四氢呋喃作溶剂, 在强碱氨基锂(LiHMDS)作用下合 成了 7 个 2-氨基苯并啞唑衍生物，产率 78\% 96\% (Eq. 4). 推测其机理可能是: 在氨基锂作用下, 邻氨基苯酚 与 NCTS 首先反应形成 15 ; 然后通过分子内消除 $N$-苯 基对甲苯磺酰胺后形成中间体 16, 接着 16 通过环合反 应形成 17; 最后通过分子内 $\mathrm{H}$ 的重排生成目标产物 2 (Scheme 6). 该反应虽然反应产率高, 但原料 NCTS 价 格较贵, 且反应中需要强碱氨基锂.

2014 年, Chien 等 ${ }^{[31]}$ 首先以邻羟基苯腈与 50\% 羟胺 
<smiles>[R]=Cc1ccc(N)c(O)c1</smiles>

$\underset{\text { THF, } 5^{\circ} \mathrm{C}-\text { r.t. }}{\stackrel{\text { LiHMDS }}{\longrightarrow}}$

$\mathrm{R}=\mathrm{H}, \mathrm{CH}_{3}, \mathrm{OCH}_{3}, \mathrm{NO}_{2}, \mathrm{Cl}, \mathrm{Br}, \mathrm{C}_{6} \mathrm{H}_{5}$, etc.<smiles></smiles>

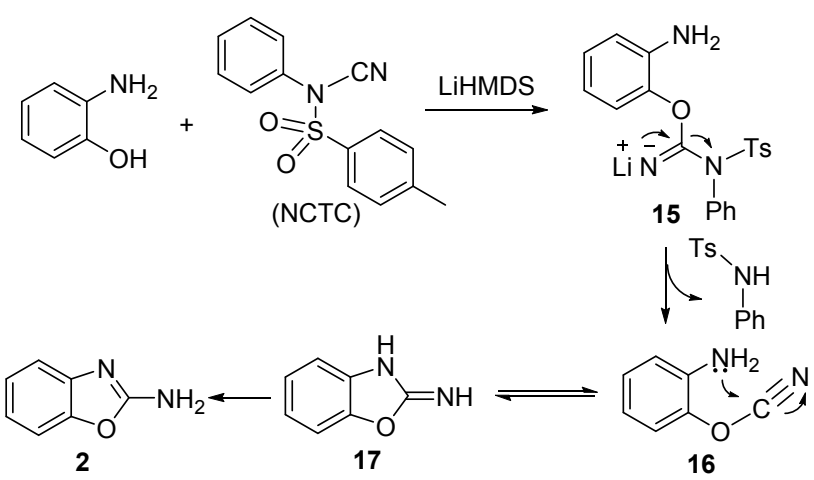

图式 6 合成化合物 2 的反应机理

Scheme 6 Reaction mechanism of synthesis of compound 2

水溶液在乙醇中回流 $3 \mathrm{~h}$ 得到邻羟基苯胺的衍生物 $\mathbf{1 7}$; 然后 17 在对甲苯磺酰氯( $(\mathrm{TsCl})$ 和二异丙基乙胺(DIPEA) 的作用下, 在 $\mathrm{CH}_{2} \mathrm{Cl}_{2}$ 溶液中回流 $3 \mathrm{~h}$ 得到 2-胺基苯并噁 唑衍生物 2, 产率达到 67\% (Eq. 5). 该反应具有反应条 件温和, 副产物少等特点, 并且该方法能应用于 2-取代 苯并咪唑衍生物的合成.<smiles>N/C(=N\O)c1ccccc1O</smiles>

17<smiles>Nc1nc2ccccc2o1</smiles>

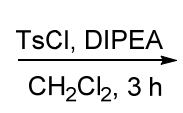

2015 年, Naga Raju 等 ${ }^{[32]}$ 以邻羟基苯胺为原料, 在 $\mathrm{KOH}$ 碱性条件下, 在乙醇溶剂中与 $\mathrm{CS}_{2}$ 回流反应 $8 \mathrm{~h}$ 得 到 2-統基苯并噁唑; 然后与 $\mathrm{PCl}_{5}$ 在干燥的甲苯溶液中 $120{ }^{\circ} \mathrm{C}$ 反应 $3 \mathrm{~h}$ 得到 2-氯代苯并噁唑衍生物 $18 ; 18$ 与取 代的哌嗪在还原锌粉存在下，微波辅助反应 $65 \mathrm{~s}$ 得到 12 个 2-胺基苯并噁唑衍生物 19, 产率达到 55\% 75\% (Scheme 7).

\section{2 以 2-取代苯并噁唑为原料合成 2-胺基苯并噁 唑衍生物}

以 2-取代苯并噁唑为原料, 通过与胺类化合物发生 亲核取代反应是合成 2-胺基苯并噁唑衍生物另一种途 径. 通常 2-取代苯并噁唑包括 $\mathrm{Cl}(\mathrm{Br}), \mathrm{SH}, \mathrm{SCH}_{3}$ 和 $\mathrm{OPh}$ 等. 例如, 2014 年 Nageswar 等 ${ }^{[33]}$ 使用可循环利用的 $\mathrm{Ru} / \mathrm{C}$ 作为催化剂, 甲酰胺、二级胺、胺基氰和二烷氧基
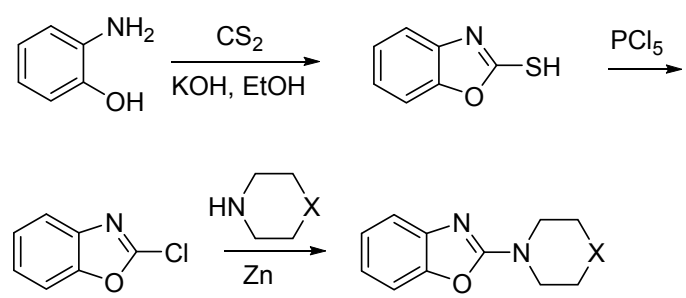

18

19

图式 7 化合物 19 的合成路线

Scheme 7 Synthetic route of compound 19

甲基胺分别与 2-氯(溴)苯并噁唑在 $\mathrm{LiO} t$-Bu 碱作用下, 在 $80{ }^{\circ} \mathrm{C}$ 温度下反应 $8 \mathrm{~h}$, 得到一系列 2-胺基苯并噁唑衍 生物，产率达到 58\% 89\% (Scheme 8). 该方法的特点 是可以使用不同的胺类化合物为胺源，且催化剂可以重 复利用.
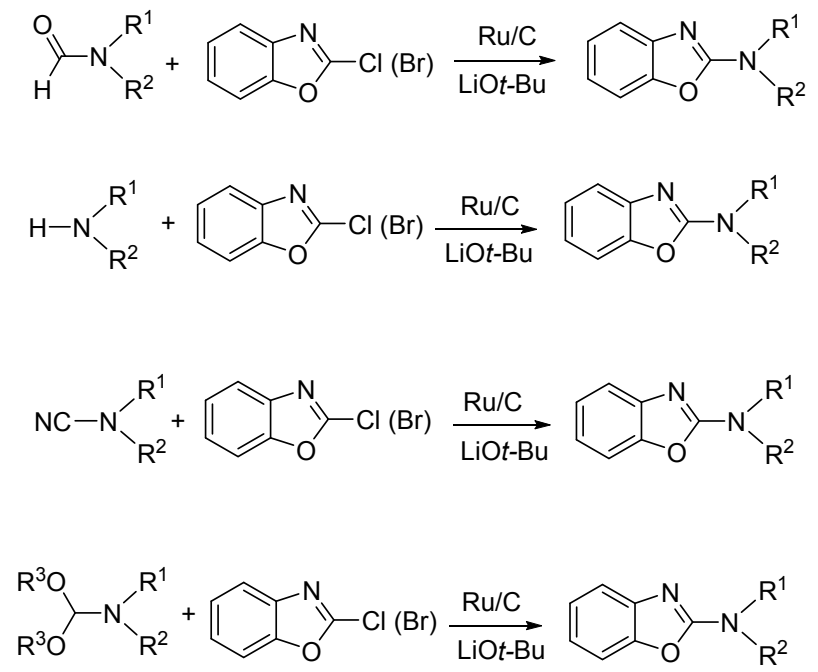

$\mathrm{R}^{1}=\mathrm{R}^{2}=\mathrm{H}, \mathrm{CH}_{3}, \mathrm{C}_{2} \mathrm{H}_{5}, \mathrm{CH}\left(\mathrm{CH}_{3}\right)_{2}$, etc.

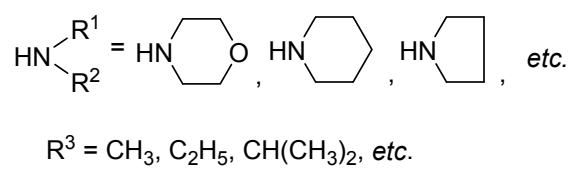

图式 8 2-胺基苯并噁唑衍生物的合成路线

Scheme 8 Synthetic route of 2-aminobenzoxazole derivatives

1984 年 Yamato 等 ${ }^{[34]}$ 以 5-氯-2-甲硫基苯并噁唑与二 级胺为原料, 加热到 $20 \sim 150{ }^{\circ} \mathrm{C}$, 合成了 8 个 2-胺基苯 并噁唑衍生物，产率为 $14 \% \sim 96 \%$; 并且该方法适用于 5-氯-2-甲硫基苯并噁唑与一级胺的反应，合成 5 个目标 产物, 产率为 $81 \% \sim 89 \%$ (Scheme 9). 其反应机理可能 为 5-氯-2-甲硫基苯并噁唑与二级胺首先发生亲核加成 反应得到中间体 20; 如果下一步 $\mathrm{C}-\mathrm{S}$ 键断裂, 将会生 成目标产物 21, 如果 $\mathrm{C}-\mathrm{O}$ 键断裂将会生成中间体，发 生分子内的亲核取代生成目标产物 21. 


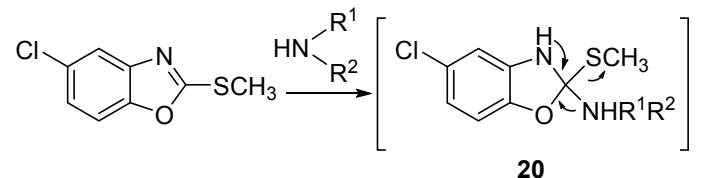<smiles>[R1]N([R])c1nc2cc(Cl)ccc2o1</smiles>

$\mathrm{R}^{1}=\mathrm{H}, \mathrm{CH}_{2} \mathrm{CH}_{2} \mathrm{OCH}_{2} \mathrm{CH}_{2}, \mathrm{PhCH}_{2}$, Ph, etc. $\mathrm{R}^{2}=\mathrm{CH}_{3}, n-\mathrm{Bu}, \mathrm{CH}_{2} \mathrm{Ph}, \mathrm{CH}_{2} \mathrm{CH}_{2} \mathrm{CH}_{2} \mathrm{OH}, \mathrm{Ph}, \mathrm{CH}_{2} \mathrm{Ph}, \mathrm{CH}(\mathrm{Cl}) \mathrm{Ph}$, etc.

图式 9 化合物 21 的合成路线

Scheme 9 Synthetic route of compound 21

1996 年, Saladino 等 ${ }^{[35]}$ 以硫代酰胺和胺类化合物为 原料, $\mathrm{O}_{3}$ 为氧化剂, 于室温下在 $\mathrm{CH}_{2} \mathrm{Cl}_{2}$ 中反应得到 2-胺 基苯并噁唑衍生物(Eq. 6).

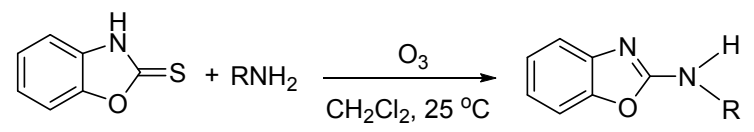

$\mathrm{R}=\mathrm{H}, \mathrm{CH}_{3}$, etc.

1994 年, Kövér 等 ${ }^{\left[{ }^{[6]}\right.} 6$-氯-2-苯氧基苯并噁唑衍生物 为原料, 在乙腈溶液中, 室温下与一级胺反应 $1 \sim 20$ $\min$ 得到 10 个 2-胺基苯并㤠唑衍生物, 产率为 $90 \%$ 98\% (Eq. 7). 该方法具有反应条件温和，反应时间短和 产率高等特点.

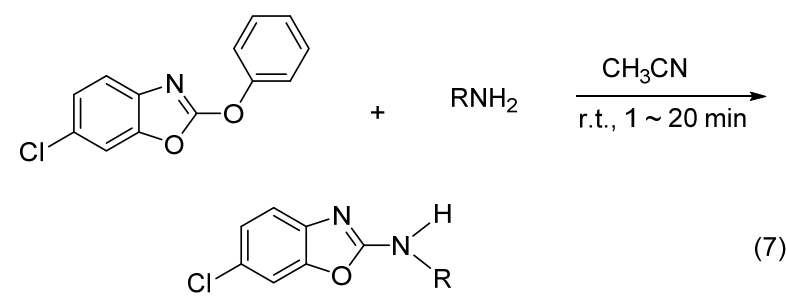

$\mathrm{R}=\mathrm{CH}\left(\mathrm{CH}_{3}\right)_{2}, \mathrm{CH}_{2}\left(\mathrm{CH}_{2}\right)_{2} \mathrm{CH}_{3}, \mathrm{CH}_{2} \mathrm{C}_{6} \mathrm{H}_{5}$, etc.

\section{3 以苯并噁唑为原料通过 2 位 C-H 键直接官 能团化合成 2-胺基苯并噁唑衍生物}

近年来, 通过取代苯并噁唑 2 位 $\mathrm{C}-\mathrm{H}$ 键直接官能 团化构建 $\mathrm{C}-\mathrm{N}$ 键合成 2-胺基苯并噁唑衍生物的催化反 应相继被报道, 主要是在金属或非金属催化下, 分别以 一级胺、二级胺、三级胺、甲酰胺和其它含氮化合物为 胺源对苯并噁唑进行直接胺基化.

\section{1 以一级胺为胺源合成 2-胺基苯并噁唑衍生物}

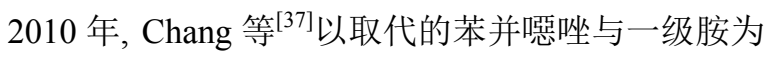
原料, $\mathrm{Mn}(\mathrm{OAc})_{2}$ 为催化剂, $\mathrm{T}-\mathrm{HYDRO}$ 为氧化剂, $\mathrm{CH}_{3} \mathrm{CN}$ 为溶剂, 在醋酸环境里 $70{ }^{\circ} \mathrm{C}$ 下反应 $12 \mathrm{~h}$ 得到 5 个 2-胺 基苯并噁唑衍生物, 产率为 $25 \% \sim 75 \%$ (Eq. 8).

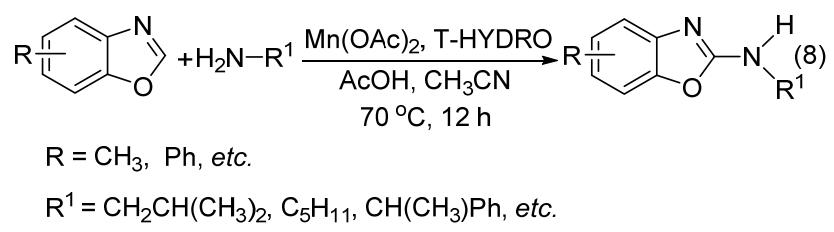

2015 年, $\mathrm{Cai}$ 等 ${ }^{[38]}$ 以 $\mathrm{CuCl}$ 为催化剂, 过氧叔丁醇 (TBHP) 为氧化剂, $\mathrm{AcOH}$ 为辅助剂, 以甲苯为溶剂, 取 代苯并噁唑与一级胺在 $80{ }^{\circ} \mathrm{C}$ 下反应 $16 \mathrm{~h}$, 得到 10 个 2胺基苯并噁唑衍生物，产率为 72\% 92\% (Eq. 9). 5-硝 基苯并噁唑、苯并噻唑和苯并咪唑与苄胺在该条件下不 反应. 但是, 部分二级胺和三级胺在该条件下能与苯并 噁唑反应生成目标产物，且具有较好的产率 $24 \% \sim 94 \%$.

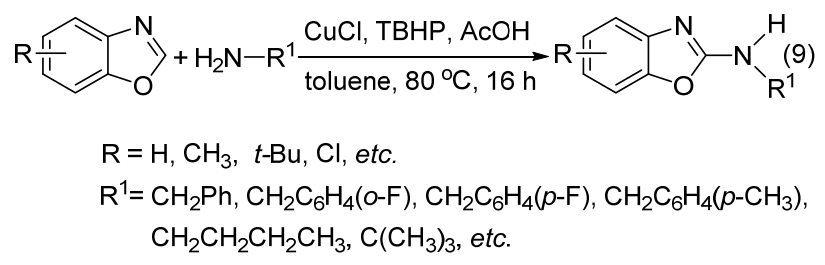

\section{2 以二级胺为胺源合成 2-胺基苯并噁唑衍生物}

\subsection{1 过渡金属催化下苯并噁唑 $\mathrm{C}(2)$ 直接胺基化}

过渡金属催化剂是有机合成中常用的金属催化剂, 凭借其优异的催化性能成为有机反应催化剂的主流. 近 年来, 通过过渡金属催化实现苯并噁唑 $\mathrm{C}(2)$ 直接胺基化 已经被发现. 例如, 2010 年, Chang 等 ${ }^{[39]}$ 以取代的苯并噁 唑与二级胺为原料, $\mathrm{Co}(\mathrm{OAc})_{2}$ 或 $\mathrm{Mn}(\mathrm{OAc})_{2}$ 为催化剂, $\mathrm{T}-\mathrm{HYDRO}$ 为氧化剂, 在醋酸的 $\mathrm{CH}_{3} \mathrm{CN}$ 溶液中反应 $12 \mathrm{~h}$ 得到 15 个 2-胺基苯并噁唑衍生物, 产率为 40\% 90\% (Eq. 10). 对苯并噁唑上取代基对反应的影响进行了考 察, 结果表明: 具有供电子基的苯并噁坐的产率明显增
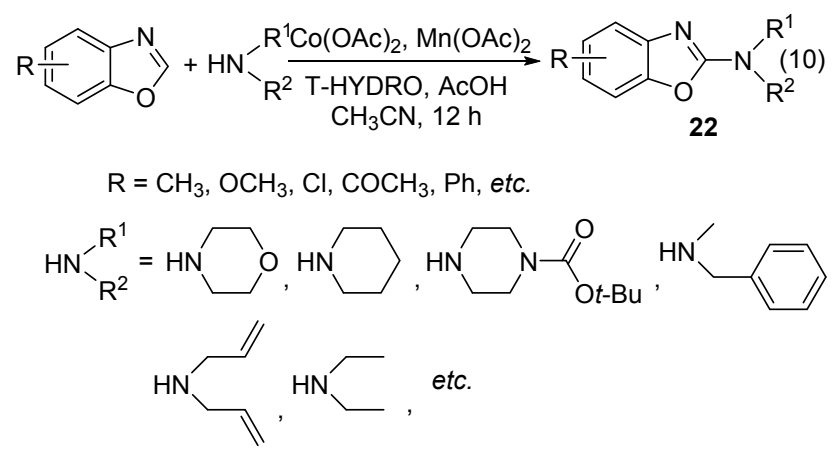
加, 5-苯基苯并噁唑与 $N$-甲基苠胺的反应产率达到 $90 \%$. 其可能的反应机理是: 苯并噁唑首先在酸性条件下形成 盐, 再与二级胺发生亲核加成反应, 脱去一分子酸; 最 后在氧化剂作用下，生成目标产物(Scheme 10).
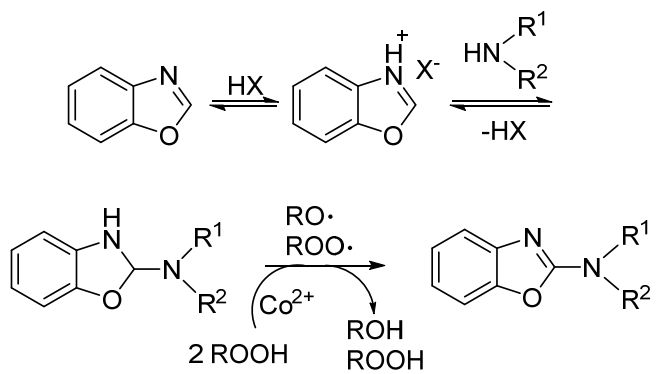

图式 10 合成 2-胺基苯并噁唑衍生物的反应机理

Scheme 10 Reaction mechanism of synthesis of 2-aminobenzoxazole derivatives

2012 年, $\mathrm{Li}$ 课题组 ${ }^{[40]}$ 以取代的苯并噁唑与二级胺为 原料, $\mathrm{Ni}_{2}(\mathrm{OAc})_{2} \bullet 4 \mathrm{H}_{2} \mathrm{O}$ 为催化剂, 过氧叔丁醇 $(\mathrm{TBHP})$ 为 氧化剂, 以乙酸作为酸性环境, 在 $70{ }^{\circ} \mathrm{C}$ 乙腈中反应 12 $\mathrm{h}$, 合成了 20 个 2-胺基苯并啞唑衍生物, 产率达 35\% $82 \%$ (Eq. 11).

$$
\begin{aligned}
& \mathrm{R}=\mathrm{CH}_{3}, t-\mathrm{Bu}, \mathrm{Cl} \text {, etc. } \\
& \mathrm{R}^{1}=\mathrm{R}^{2}=\mathrm{Et}, n-\mathrm{Bu}, n-\mathrm{Pr}, \mathrm{CH}_{2} \mathrm{CH}=\mathrm{CH}_{2} \text {, etc. } \\
& \mathrm{R}^{1}=\mathrm{CH}_{3} ; \mathrm{R}^{2}=\mathrm{C}_{6} \mathrm{H}_{5}, \mathrm{C}_{6} \mathrm{H}_{5} \mathrm{CH}_{2}, o-\mathrm{Cl}_{-} \mathrm{C}_{6} \mathrm{H}_{4}, \text { etc. }
\end{aligned}
$$

2012 年, 蔡双飞等 ${ }^{[41]}$ 以苯并恶唑和胺为底物, 以钴 纳米晶(或钴粉)为催化剂, 以 $70 \%$ 叔丁基过氧化氢 $(\mathrm{TBHP})$ 水溶液为氧化剂, 以 $\mathrm{AcOH}$ 为添加剂; 在室温空 气氛围下, 乙腈为溶剂反应, 合成了一系列 2-胺基苯并 啞唑衍生物, 产率 54\% 93\% (Eq. 12). 该方法具有反 应条件温和、原料和催化剂低廉、高效等优点, 避免了 已有合成路线中需要高温、昂贵的金属催化剂或配体等 缺点.

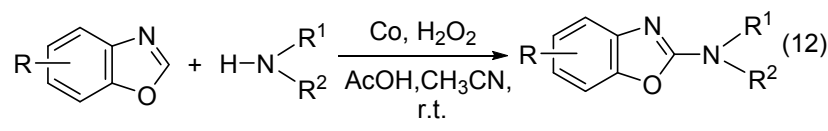
$\mathrm{R}=\mathrm{H}, \mathrm{CH}_{3}, \mathrm{OCH}_{3}, \mathrm{Cl}, \mathrm{Ph}, \mathrm{COCH}_{3}$, etc.

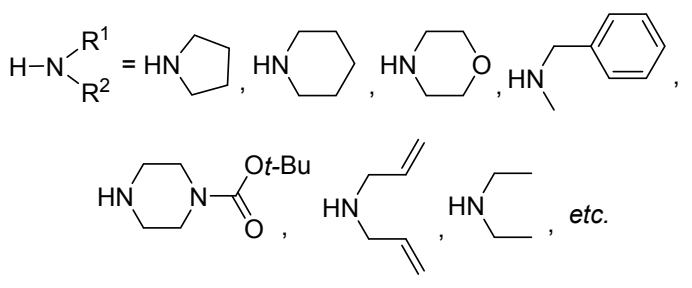

2011 年, $\mathrm{Li}$ 等 ${ }^{[42]}$ 以取代苯并啞唑与二级胺为原料, $\mathrm{Cu}(\mathrm{OAc})_{2} \cdot \mathrm{H}_{2} \mathrm{O}$ 为催化剂, $\mathrm{PhCOOH}$ 为辅助剂, 在氧气的 氛围中, 乙腈为溶剂, 在 $70{ }^{\circ} \mathrm{C}$ 下反应 $12 \mathrm{~h}$, 合成了 16 个 2-胺基苯并噁唑衍生物，产率为 20\% 94\% (Eq. 13). 该合成方法不适合以一级胺作为胺源合成 2-胺基苯并 啞唑衍生物.

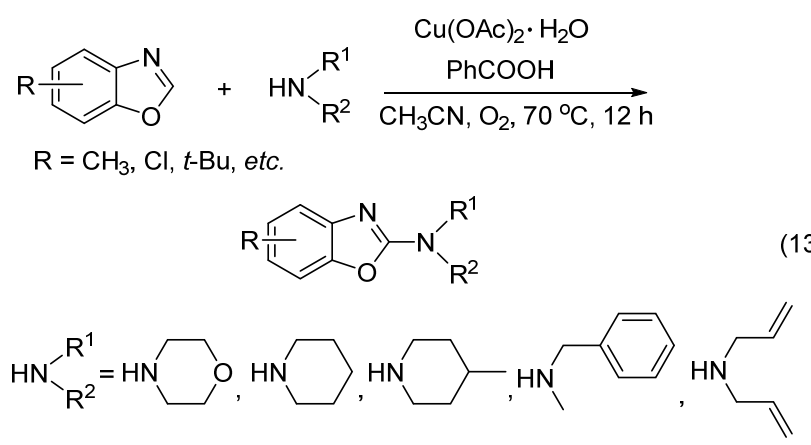

2009 年, Moti 等 ${ }^{[43,44]}$ 以取代苯并噁唑与二级胺为原 料, $\mathrm{Cu}(\mathrm{OAc})_{2}$ 为催化剂, $\mathrm{PPh}_{3}$ 作为碱, 在 $101 \mathrm{kPa}$ 氧气条 件下, 以二氧六环为溶剂 $140{ }^{\circ} \mathrm{C}$ 条件下反应 $20 \mathrm{~h}$, 合成 了 4 个 2-胺基苯并噁唑衍生物，产率为 47\% 71\% (Eq. 14). 该方法不仅可以合成2-胺基苯并噁唑，而且可以用 来合成 2-胺基苯并噻唑等衍生物. 但是该方法反应温度 高，且反应时间长.

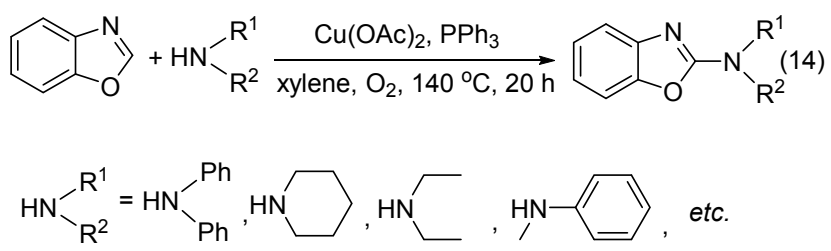

2014 年, Ghosh 等 ${ }^{[45]}$ 以多孔性 $\gamma-\mathrm{MnO}_{2}$ 为催化剂, $\mathrm{O}_{2}$ 为氧化剂, 取代苯并噁唑与二级胺在 $\mathrm{CH}_{3} \mathrm{CN}$ 溶剂中 $50{ }^{\circ} \mathrm{C}$ 反应 $24 \mathrm{~h}$, 得到 19 个 2-胺基苯并噁唑衍生物, 产 率为 $30 \% \sim 83 \%$ (Eq. 15). 该方法不仅适用于二级胺作 为胺源对苯并噁唑直接进行胺基化, 而且也适用于一级

$$
\text { }
$$

$\mathrm{R}=\mathrm{CH}_{3}, \mathrm{Cl}$, etc.

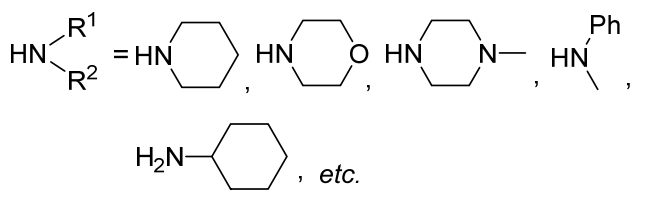


胺，甚至杂环芳香胺 3-胺基吡啶和含有活泼基团 $\mathrm{OH}$ 的 胺也能很好地反应. 另外, 该方法中使用的催化剂多孔 性 $\gamma-\mathrm{MnO}_{2}$ 具有重复利用性.

2013 年, Huang 等 ${ }^{[46]}$ 使用芳香醛和 $\mathrm{CuBr}_{2}$ 作为催化 体系, $\mathrm{O}_{2}$ 为氧化剂, $\mathrm{AcOH}$ 为辅助剂, 以 $\mathrm{CH}_{2} \mathrm{Cl}_{2}$ 为溶剂, 取代苯并啞唑与二级胺在室温下反应 $24 \mathrm{~h}$, 合成了 20 个 2-胺基苯并啞唑衍生物, 产率为 52\% 89\% (Eq. 16). 利用同位素标记、同位素动力学等实验对反应机理进行 了研究，揭示了该反应中有缩醛胺的形成.

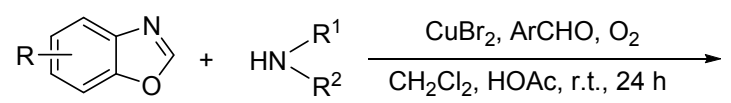
$\mathrm{R}=\mathrm{CH}_{3}, \mathrm{Cl}, t-\mathrm{Bu}, \mathrm{Ph}$, etc.

$$
\mathrm{R}
$$
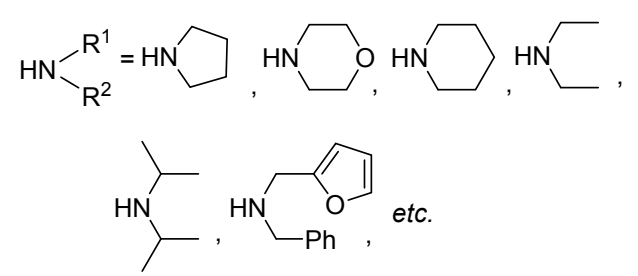

2014 年, $\mathrm{CaO}$ 等 ${ }^{[47]}$ 以 $\mathrm{CuBr}_{2}$ 为催化剂, $\mathrm{AcOH}$ 为辅助 剂, 乙腈为溶剂, 取代的苯并噁唑与二级胺在 $50{ }^{\circ} \mathrm{C}$ 条 件下反应 $12 \mathrm{~h}$, 得到 8 个 2-胺基苯并啞唑衍生物, 产率 为 $31 \% \sim 95 \%$ (Eq. 17). 该反应的机理可能是: 苯并噁 唑与铜盐首先反应形成配合物; 然后与二级胺反应形成 开环的中间体 $\mathbf{A}$; 在 $\mathrm{AcOH}$ 作用下, $\mathbf{A}$ 通过分子内的关 环反应形成中间体 $\mathbf{B}$; 最后在 $\mathrm{O}_{2}$ 作用下, 通过氧化脱氢 得到目标产物(Scheme 11). 在该反应条件下，二甲胺或 一级胺与苯并噁唑不发生反应; 苯并噻唑或苯并咪唑与 二级胺不发生反应.

$$
\text { , etc. }
$$

3.2.2 非金属催化下苯并噁唑 $\mathrm{C}(2)$ 直接胺基化

在金属催化剂蓬勃发展的同时, 催化剂的另一个分 支非金属有机催化剂引起了人们的浓厚兴趣, 越来越多 的有机分子被直接用作有机反应的催化剂. 例如, 2011

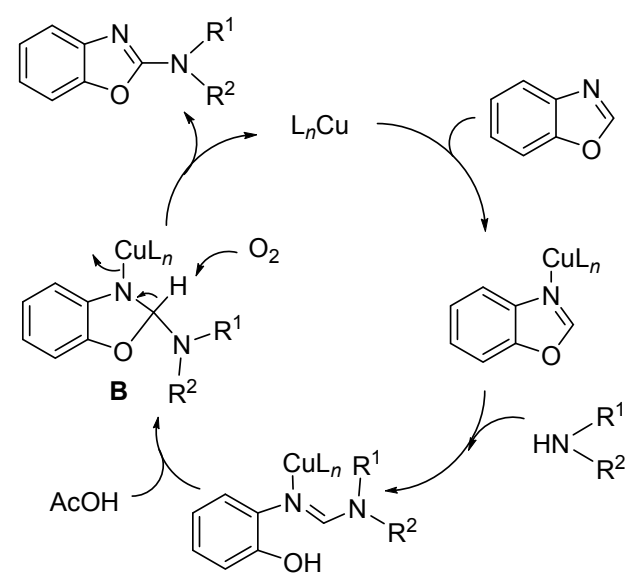

A

图式 11 合成 2-胺基苯并噁唑衍生物的反应机理 Scheme 11 Reaction mechanism of synthesis of 2-aminobenzoxazole derivatives

年, Prabhu 等 ${ }^{[48]}$ 以取代的苯并噁唑与二级胺为原料, $I_{2}$ 为 催化剂, $\mathrm{TBHP}$ 为氧化剂, $\mathrm{AcOH}$ 为辅助剂, 在室温下反 应 $12 \mathrm{~h}$ 得到 18 个 2-胺基苯并噁唑衍生物, 产率高达 $80 \% \sim 95 \%$ (Eq. 18); 该方法还能应用到苯并噁唑与一 级胺的反应，且具有较好的产率 $63 \% \sim 80 \%$. 该方法具 有反应条件温和，适用范围广，非金属为催化剂，反应 产率高等优点. 其反应机理可能是: 苯并噁唑与 $\mathrm{AcOH}$ 首先反应生成铵盐, 接着二级胺与铵盐发生亲核加成反 应生成中间体 23; 然后 23 在 $\mathrm{I}_{2}$ 作用下生成中间体 24; 最后 24 脱去小分子 HI 生成目标产物(Scheme 12).

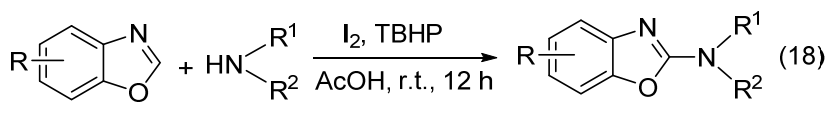
$\mathrm{R}=\mathrm{CH}_{3}, \mathrm{NO}_{2}, \mathrm{Ph}$, etc.<smiles>[R]NN1CCOCC1CNc1ccccc1</smiles>

2011 年, Nachtsheim 等 ${ }^{[49]}$ 以取代苯并噁唑与二级胺 为原料, 四正丁基碘化铵(TBAI)为催化剂, $\mathrm{AcOH}$ 为辅 助剂, $\mathrm{CH}_{3} \mathrm{CN}$ 为溶剂, 以 $30 \% \mathrm{H}_{2} \mathrm{O}_{2}$ 水溶液为氧化剂, 在 室温下反应，共合成 18 个 2-胺基苯并噁唑衍生物，产率 为 $17 \% \sim 93 \%$; 或以 $\mathrm{TBHP}$ 为氧化剂, 在 $80{ }^{\circ} \mathrm{C}$ 反应合成 目标化合物，产率 $52 \% \sim 87 \%$ (Eq. 19). 利用该反应条 件, 成功合成了具有潜在抗 HIV 和抗肿瘤活性的化合物 (Eq. 20).

2012 年, Bhanage 等 ${ }^{[50]}$ 以 $30 \% \mathrm{H}_{2} \mathrm{O}_{2}$ 水溶液为氧化 剂, 催化量的 $N$-碘代丁二酰亚胺(NIS)为催化剂, 在无 过渡金属存在的条件下，以乙腈为溶剂，取代苯并噁唑 与二级胺在室温下反应, 得到 15 个 2-胺基苯并啞唑衍 


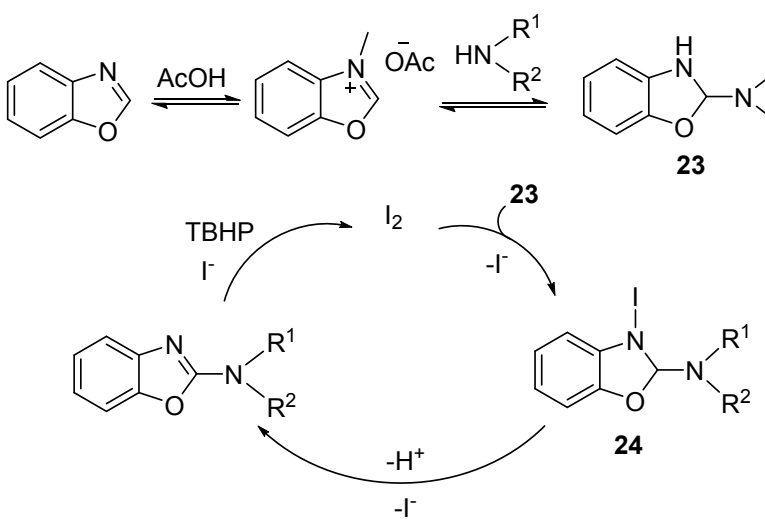

图式 12 合成 2-胺基苯并噁唑衍生物的反应机理

Scheme 12 Reaction mechanism of synthesis of 2-aminobenzoxazole derivatives

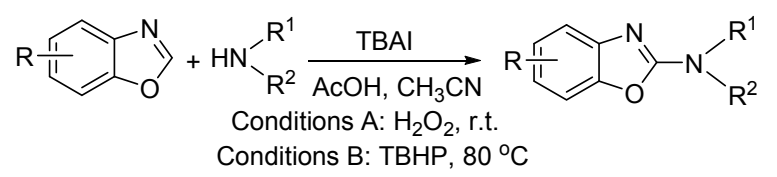

$\mathrm{R}=\mathrm{CH}_{3}, \mathrm{Cl}, \mathrm{NO}_{2}, t-\mathrm{Bu}$, etc.

$$
\text { , etc. }
$$

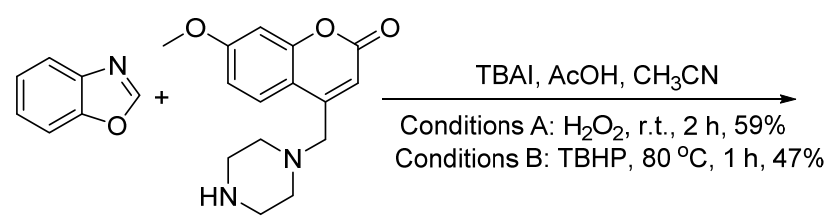<smiles>COc1ccc2c(CN3CCN(c4nc5ccccc5o4)CC3)cc(=O)oc2c1</smiles>

生物, 产率为 $80 \% \sim 98 \%$ (Eq. 21). 该反应不仅适用于 二级胺为胺源对苯并啞唑进行直接胺基化, 而且一级脂 肪胺也能反应，且具有较好的产率 $81 \% \sim 82 \%$.

2014 年, Zeng 等 ${ }^{[51]}$ 利用电化学的方法, 使取代的苯 并噁唑与二级胺的反应在一个无隔膜的电解槽 (GC 为 阳极, $\mathrm{Fe}$ 为阴极, $6 \mathrm{~mA} / \mathrm{cm}^{2}$ 电流强度) 中进行. 以 $n-\mathrm{Bu}_{4} \mathrm{NI}$ 为催化剂, $\mathrm{AcOH}$ 为辅助剂, 在乙腈溶液中室温 下反应, 得到 16 个 2-胺基苯并噁唑衍生物, 产率为 30\% 97\% (Eq. 22). 其可能的机理是: 苯并噁唑首先在 $\mathrm{AcOH}$ 条件下发生质子化得到 25; 25 与二级胺反应生成 开环的化合物 26; 26 发生分子内的亲核加成得到闭环的

$$
\text { (21) }
$$

化合物 27; $\mathrm{X}^{-}$在电解槽的阳极反应生成 $\mathrm{X}^{+}$, 然后与 27 反应，失去一个 $\mathrm{H}^{+}$得到 $28 ; 28$ 脱去一个 $\mathrm{HX}$ 分子得到目 标化合物(Scheme 13). 该方法具有反应底物作为阳极, 无需向体系中加入氧化剂, 不需要向电解槽中加入电解 液, 仅需要催化量的 $n-\mathrm{Bu}_{4} \mathrm{NI}$ 为催化剂等优点; 但是该 方法不适合利用一级胺或琥珀酰亚胺、邻苯二甲酰亚胺 等二级胺为胺源来合成 2-胺基苯并噁唑衍生物.

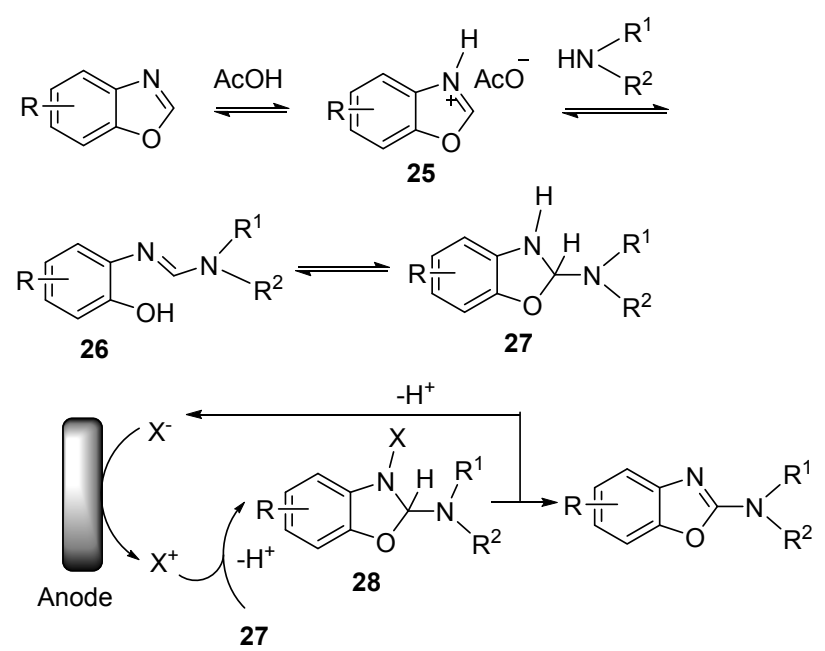

图式 13 合成 2-胺基苯并噁唑衍生物的反应机理

Scheme 13 Reaction mechanism of synthesis of 2-aminobenzoxazole derivatives

3.2.3 通过开环和关环分步反应实现苯并噁唑 $C(2)$ 的胺基化

2011 年, Chang 等 ${ }^{[52]}$ 以取代的苯并噁唑与二级胺为 原料，在无氧化剂或催化剂的条件下，反应后首先得到 
开环产物; 然后在室温下, 以高价碘化物 $\mathrm{Ph}(\mathrm{OAc})_{2}$ 为氧 化剂, 通过关环反应得到 10 个 2-胺基苯并噁唑衍生物, 产率为 $61 \% \sim 90 \%$ (Eq. 23). 该方法通过二步反应一锅 煮的方法, 不需分离中间产物, 提高了反应效率; 不使 用金属催化剂或氧化剂, 酸或碱等辅助剂, 反应条件温 和. 但是该方法不适合以一级胺为胺源来合成 2-胺基苯 并噁唑衍生物.

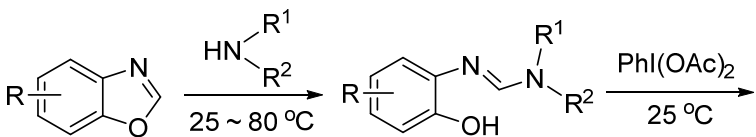

$$
\begin{aligned}
& \text { L }
\end{aligned}
$$

$\mathrm{R}=\mathrm{H}, \mathrm{CH}_{3}, \mathrm{OCH}_{3}, \mathrm{Cl}, \mathrm{NO}_{2}, \mathrm{Ph}$, etc.

$$
\text { , etc. }
$$

2011 年, Studer 等 ${ }^{[53]}$ 以二级胺为胺源, 首先以三氟 甲磺酸 $(\mathrm{TfOH})$ 或三氟甲磺酸钪 $\left[\mathrm{Sc}(\mathrm{OTf})_{3}\right]$ 为辅助剂, 2,2,6,6-四甲基哌啶(TEMP)用作碱, 在乙腈溶液中室温 或 $60{ }^{\circ} \mathrm{C}$ 下与苯并噁唑反应 $4 \mathrm{~h}$; 然后在 2,2,6,6-四甲基 哌啶- $N$-氧自由基四氟硼酸盐 $\left(\mathrm{TEMPO}^{+} \mathrm{BF}_{4}{ }^{-}\right.$) 和 TEMP 作用下, 反应 $10 \mathrm{~min}$, 得到 22 个 2 -胺基苯并噁唑衍生 物, 产率为 $26 \% \sim 96 \%$ (Eq. 24). 反应机理可能是: 在辅 助剂 $\mathrm{TfOH}$ 或 $\mathrm{Sc}(\mathrm{OTf})_{3}$ 和碱 TEMP 共同作用下, 苯并噁 唑首先与二级胺反应生成开环产物 $\mathbf{2 9}$, 然后在 TEMP$\mathrm{O}^{+} \mathrm{BF}_{4}{ }^{-}$作用下, 生成中间体 $\mathbf{3 0}, \mathbf{3 0}$ 发生分子内的加成反 应得到闭环的中间体 31; 31 在 $\mathrm{TEMPO}^{+}-\mathrm{BF}_{4}{ }^{-}$作用下生 成目标产物 7 (Scheme 14). 该反应具有反应条件温和, 产率高, 底物适用范围广等特点.

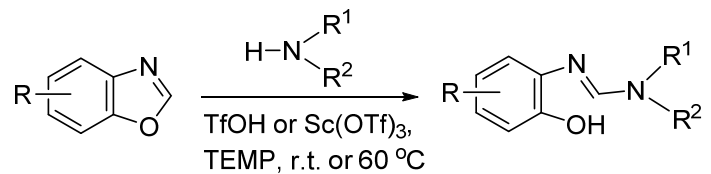

$$
\underset{T E M P, 10 \mathrm{~min}}{\stackrel{\mathrm{TEMPO}^{+} \mathrm{BF}_{4}^{-}}{\longrightarrow}}
$$

$\mathrm{R}=\mathrm{H}, \mathrm{OCH}_{3}, \mathrm{Cl}, \mathrm{Ph}$, etc.

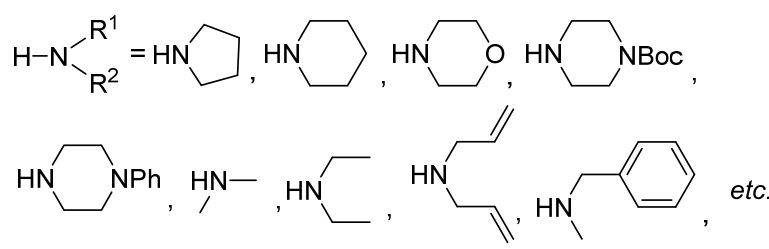

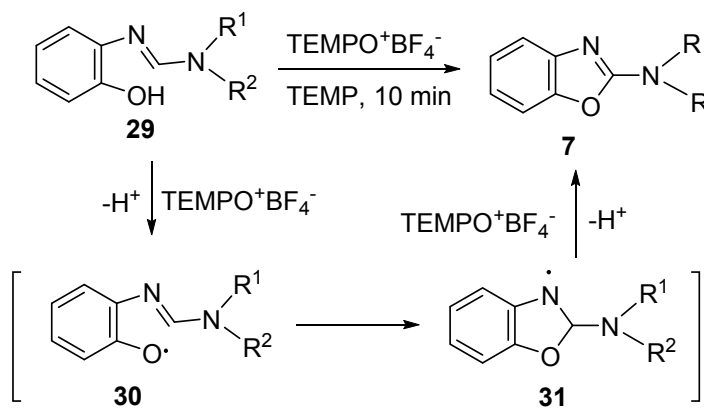

图式 14 合成 2-胺基苯并噁唑衍生物的反应机理

Scheme 14 Reaction mechanism of synthesis of 2-aminobenzoxazole derivatives

2013 年, Bhanage 等 $^{[54]}$ 以取代苯并啞唑与二级胺为 原料合成了 13 个 2-胺基苯并噁唑衍生物, 该反应分两 步进行，第一步是苯并噁唑与二级胺反应生成开环产 物; 第二步以 2 -碘酰基苯甲酸(IBX)为氧化剂, 在室温 下反应 5 30 min, 通过分子内亲核加成反应得到目标 产物，产率达 $80 \% \sim 95 \%$ (Eq. 25). 在该反应中，反应底 物适用范围广，不仅环状的二级胺能够反应，而且非环 状和带有其它官能团的脂肪胺也能参与反应，且具有较 高的产率. 该方法具有反应条件温和、反应时间短、产 率高等特点.

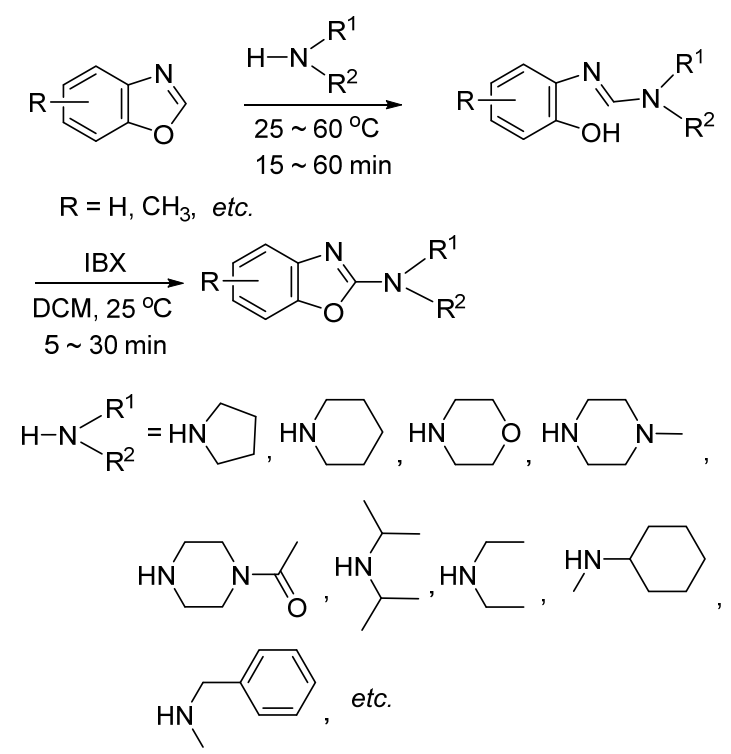

2013 年, Yotphan 等 ${ }^{[55]}$ 以 $\mathrm{I}_{2}$ 为氧化剂, 叔丁醇锂 $(\mathrm{LiO} t-\mathrm{Bu})$ 为碱, $\mathrm{THF}$ 为溶剂, 取代苯并噁唑与二级胺在 室温下反应 $1 \mathrm{~h}$, 得到 9 个 2-胺基苯并噁唑衍生物，产率 为 $64 \% \sim 98 \%$ (Eq. 22). 可能的反应机理是：苯并噁唑 在 $\mathrm{LiO} t-\mathrm{Bu}$ 和 $\mathrm{I}_{2}$ 共同作用下，生成中间体 2-碘代苯并噁 唑; 然后与二级胺反应，通过分子间的亲核取代反应得 到目标产物 2-胺基苯并噁唑衍生物(Scheme 15). 该方法 对反应底物具有广泛的适用性，不仅二级胺能够反应， 而且一级脂肪胺和芳香胺均能参与反应，产率为 $45 \%$ 66\%; 另外, 此方法也能应用到苯并噻唑、苯并咪唑等 
唑类化合物的胺基化.

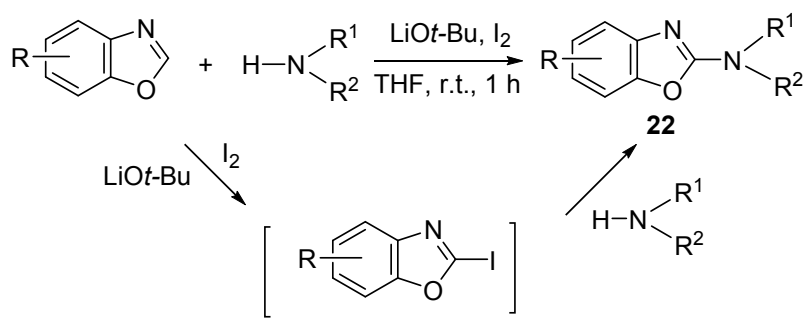

$\mathrm{R}=\mathrm{H}, \mathrm{Cl}$, etc.

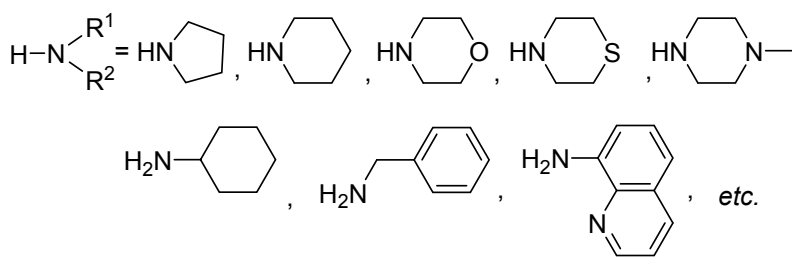

图式 15 合成 2-胺基苯并噁唑衍生物的反应机理

Scheme 15 Reaction mechanism of synthesis of 2-aminobenzoxazole derivatives

2013 年, Sun 等 ${ }^{[56]}$ 通过一锅煮的方法, 以取代苯并 噁唑与二级胺为原料, 首先在室温或 $80{ }^{\circ} \mathrm{C}$ 下反应生成 开环产物, 然后以 $\mathrm{FeCl}_{2}$ 为催化剂, $30 \% \mathrm{H}_{2} \mathrm{O}_{2}$ 水溶液为 氧化剂, 在室温下反应 $5 \mathrm{~min}$ 得到 18 个 2-胺基苯并噁唑 衍生物, 产率达 $86 \% \sim 97 \%$ (Scheme 16). 反应可能的反 应机理是: 苯并噁唑首先与二级胺反应生成开环产物 32, 32 与羟基自由基反应生成中间体 33, 33 发生分子内 的加成生成中间体 34, 34 在羟基自由基作用下脱去 $\mathrm{H}_{2} \mathrm{O}$ 分子, 生成目标产物(Scheme 17). 该方法具有反应条件 温和、催化剂和氧化剂环境友好、反应时间短、产率高 等优点.

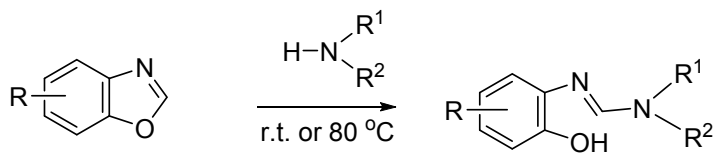

$\mathrm{R}=\mathrm{H}, \mathrm{OCH}_{3}, \mathrm{Cl}$, etc.
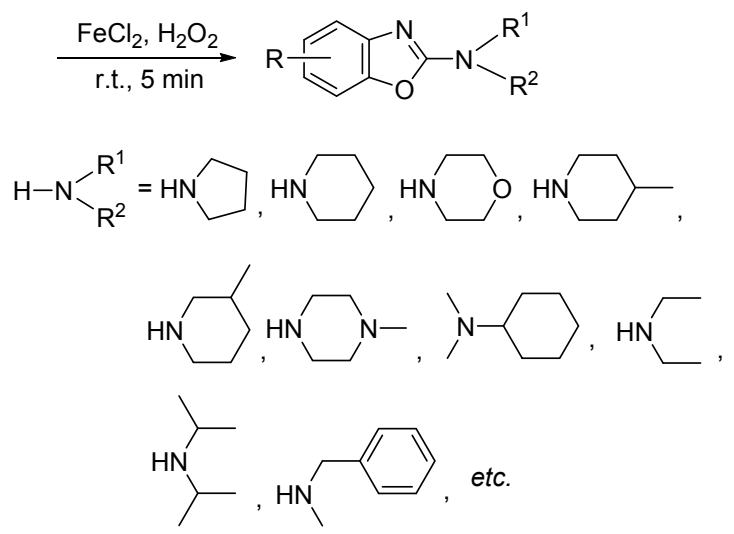

图式 16 2-胺基苯并噁唑衍生物的合成路线

Scheme 16 Synthetic route of 2-aminobenzoxazole derivatives

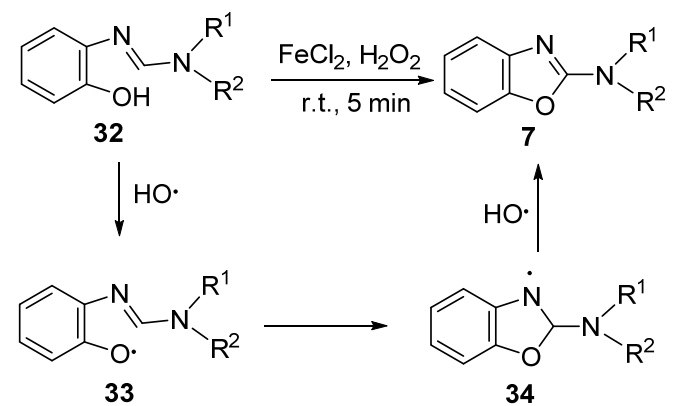

图式 17 合成 2-胺基苯并噁唑衍生物的反应机理 Scheme 17 Reaction mechanism of synthesis of 2-aminobenzoxazole derivatives

2014 年, Sun 等 ${ }^{[57]}$ 以 $N$-溴代丁二酰亚胺(NBS)为氧 化剂, KOAc 为碱, 二氧六环与水为共溶剂, 取代苯并噁 唑与二级胺反应生成 18 个 2-胺基苯并噁唑衍生物, 产 率为 $82 \% \sim 93 \%$ (Scheme 18). 反应机理可能是: 第一步 苯并噁唑与二级胺反应生成开环产物 $\mathbf{3 5}$; 第二步是 35 与 NBS 反应形成中间体 36, 36 脱去一分子丁二酰亚胺 生成中间体 37,38 是 36 的共振式; 通过分子内的亲核加 成得到中间体 39, 在 KOAc 作用下, 39 脱去一分子 $\mathrm{HBr}$ 得到目标产物(Scheme 19). 该方法具有反应时间短、使 用非金属氧化剂、反应条件温和、产率高等特点.
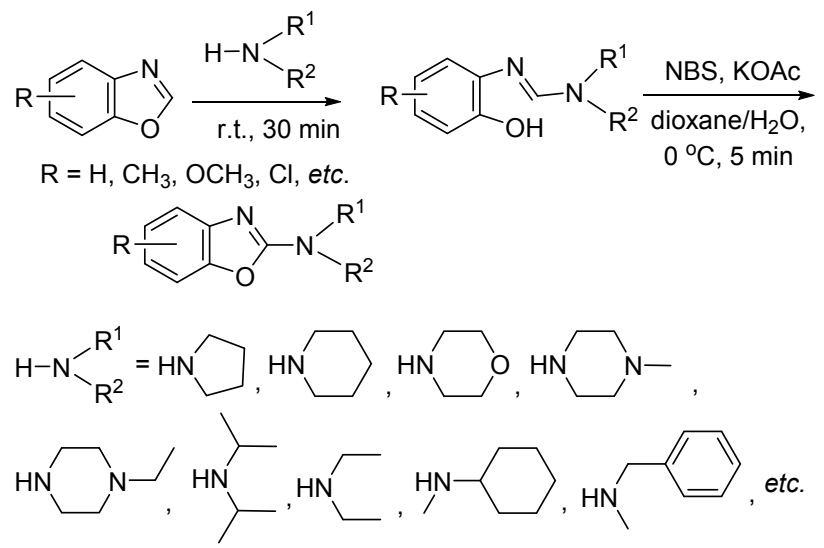

图式 18 2-胺基苯并啞唑衍生物的合成路线 Scheme 18 Synthetic route of 2-aminobenzoxazole derivatives

\subsection{4 光催化对苯并噁唑 $\mathrm{C}(2)$ 直接胺基化}

近年来，光催化在有机合成领域得到广泛的关注， 该方法利用金属络合物或有机染料吸收可见光生成易 于进行单电子转移的激发态中间体，从而对反应物进行 活化, 通过单电子转移与反应物作用生成各种活性中间 体从而参与到不同的反应中. 光催化氧化还原反应为自 由基的产生提供了一种绿色方法，由于该反应具有无 毒、安全、稳定性好、催化活性高、见效快和能耗低等 优点，因此该方法受到广泛的关注 ${ }^{[58 \sim 61]}$.

2014 年, Xiao 等 ${ }^{\left[{ }^{[2]}\right.}$ 以取代苯并噁唑与二级胺为原 
<smiles>[R]N([R])/C=N/c1ccccc1O</smiles><smiles>[R]N([R])/C=N/[B]N1C(=O)CCC1=O</smiles><smiles>CC(C)C1C(C)CC1C</smiles><smiles></smiles>

37<smiles>[R][N+](C)=CN(Br)c1ccccc1O</smiles>

38<smiles>[R]N([R])c1nc2cc(C(C)(C)C)ccc2o1</smiles>

39

图式 19 合成 2-胺基苯并噁唑衍生物的反应机理

Scheme 19 Reaction mechanism of synthesis of 2-aminobenzoxazole derivatives

料, $N$-氯代丁二酰亚胺(NCS)辅助剂, 室温下在 $\mathrm{CH}_{2} \mathrm{Cl}_{2}$ 溶剂中反应 $3 \mathrm{~h}$; 然后加入催化剂 $\left[\operatorname{Ir}(\mathrm{dtbpy})(\mathrm{ppy})_{2}\right] \mathrm{PF}_{6}$ 和 碱 $\mathrm{Ph}_{3} \mathrm{~N}$, 用 $3 \mathrm{~W}$ 蓝色 LED 灯照射 $60 \mathrm{~h}$, 得到 15 个 2胺基苯并噁唑衍生物, 产率 34\% 75\% (Scheme 20). 可 能的反应机理是: 在 NCS 和[Ir]/LEDs 作用下, 二级胺生 成胺基自由基; 然后与苯并噁唑反应生成中间体 40, 中 间体 40 失去一个电子并失去一个质子后, 得到目标产 物. 该方法的底物的适用范围广, 反应条件温和, 所用 试剂对环境友好，但是反应时间长，产率适中.

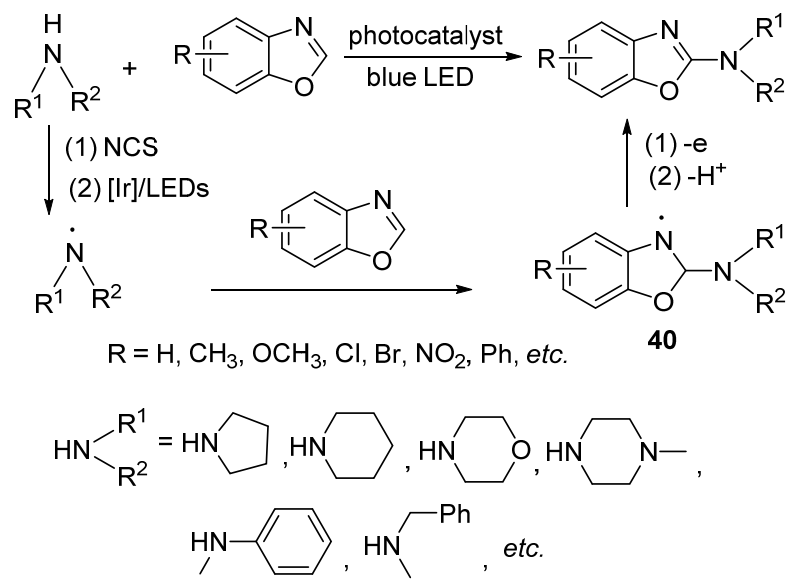

图式 20 2-胺基苯并噁唑衍生物的合成路线与反应机理

Scheme 20 Synthetic route and reaction mechanism of 2-aminobenzoxazole derivatives

\section{3 以三级胺为胺源合成 2-胺基苯并噁唑衍生物}

3.3.1 过渡金属催化下苯并噁唑 $\mathrm{C}(2)$ 直接胺基化 2011 年, Huang 等 ${ }^{[63]}$ 以三级胺为胺源, 在 $\mathrm{CuBr}_{2}$ 催 化下, $\mathrm{AcOH}$ 为辅助剂, 空气中的 $\mathrm{O}_{2}$ 为氧化剂, 与取代
的苯并噁唑在二氧六环溶剂中 $120{ }^{\circ} \mathrm{C}$ 下反应 $16 \mathrm{~h}$, 成功 合成了 22 个 2 -胺基苯并啞唑衍生物，产率为 20\% 95\% (Eq. 26). 可能的反应机理是: 首先铜盐与空气中的 $\mathrm{O}_{2}$ 反应生成 $\left[\mathrm{CuL}^{n+1}\right]$, 与三级胺反应形成配合物 41 , 同时 在醋酸作用下脱去一个水分子; 41 发生水解, 脱去一分 子醛生成中间体 $42 ; 42$ 与苯并噁唑形成配合物 43,43 通 过脱去一个 $\mathrm{H}^{+}$和重排生成一个 $\mathrm{CH}_{3} \mathrm{COOH}$ 分子, 从而 形成中间体 44 ; 最后通过还原消除生成目标产物，同时 生成原来的催化剂铜盐(Scheme 21). 该反应原料和金 属催化剂廉价易得, 氧化剂环保经济的特点, 但是该反 应温度高, 且反应时间较长.

$$
\begin{aligned}
& \mathrm{R}=\mathrm{CH}_{3}, \mathrm{Cl}, \mathrm{Br}, \mathrm{NO}_{2}, t-\mathrm{Bu}, \mathrm{Ph} \text {, etc. } \\
& \mathrm{R}^{1}=\mathrm{R}^{2}=\mathrm{R}^{3}=\mathrm{Et}, n-\mathrm{Pr}, n-\mathrm{Bu}, i-\mathrm{Bu} \text {, Oct, Bn, etc. }
\end{aligned}
$$

\subsection{2 非金属催化下苯并噁唑 C(2)直接胺基化}

2016 年, 本课题组 ${ }^{[64]}$ 在无过渡金属催化的条件下, 以三级胺为胺源, 四丁基碘化铵(TBAI)为催化剂, 醋酸 $(\mathrm{AcOH})$ 为辅助剂, 过氧叔丁醇( TBHP) 为氧化剂, 乙腈 为溶剂, 在微波辅助下, 与取代苯并惡唑反应 $20 \mathrm{~min}$, 合成了 23 个 2 -胺基苯并啞唑衍生物，产率为 $45 \% \sim 69 \%$ (Eq. 27). 可能的反应机理是：第一步首先 TBHP 在 $\mathrm{I}^{-}$催 化下生成 $t-\mathrm{BuO}$ 自由基和 $t-\mathrm{BuO}$ 自由基; 第二步是三级 胺在 $t-\mathrm{BuO}$ 自由基作用下，生成三级胺的碳自由基 45 , 通过单电子转移得到烯胺正离子 46, 再与 $\mathrm{H}_{2} \mathrm{O}$ 加成脱 去 $\mathrm{H}^{+}$, 生成中间体胺醇 47, 47 脱去一分子醛得到二级 胺; 第三步是苯并噁唑在 $\mathrm{AcOH}$ 条件下发生质子化, 得 到质子化产物 48, 48 与二级胺发生亲核加成, 脱去一分 子 $\mathrm{AcOH}$, 生成中间体 49, 49 在 $t-\mathrm{BuO}$ 或 $t-\mathrm{BuO}$ 自由基 作用下, 生成目标产物(Scheme 22). 该方法具有反应条 件温和，反应时间短，底物的适用范围广等特点.

$\mathrm{Yu}$ 等 ${ }^{[65]}$ 以不同的三级胺与芳香醛为原料, 在类似 的条件下，合成了一系列的酰胺衍生物. 另外，在该条 件下，以苯并啞唑与三乙胺反应，合成了一个 2-胺基苯 并噁唑衍生物, 产率为 $50 \%$. 


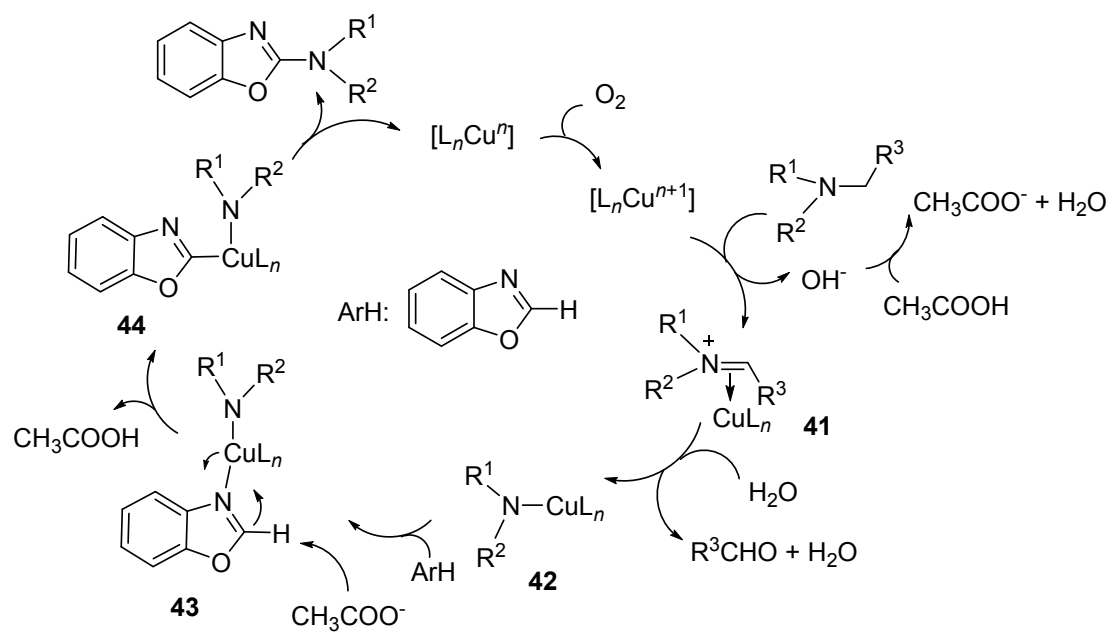

图式 21 合成 2-胺基苯并噁唑衍生物的反应机理

Scheme 21 Reaction mechanism of synthesis of 2-aminobenzoxazole derivatives

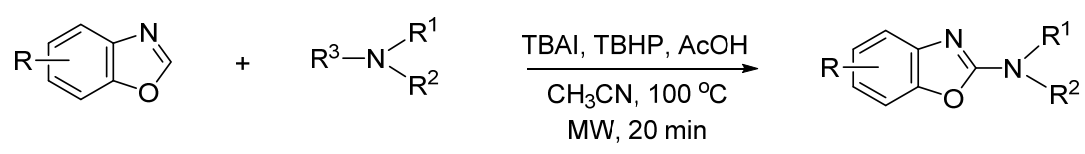

$\mathrm{R}=\mathrm{H}, \mathrm{CH}_{3}, t-\mathrm{Bu}, \mathrm{Cl}, \mathrm{NO}_{2}$, etc.

$\mathrm{R}^{1}=\mathrm{R}^{2}=\mathrm{R}^{3}=\mathrm{CH}_{3}, \mathrm{CH}_{2} \mathrm{CH}_{3}, \mathrm{CH}_{2} \mathrm{CH}_{2} \mathrm{CH}_{3}, \mathrm{CH}_{2} \mathrm{CH}_{2} \mathrm{CH}_{2} \mathrm{CH}_{3}$, etc.
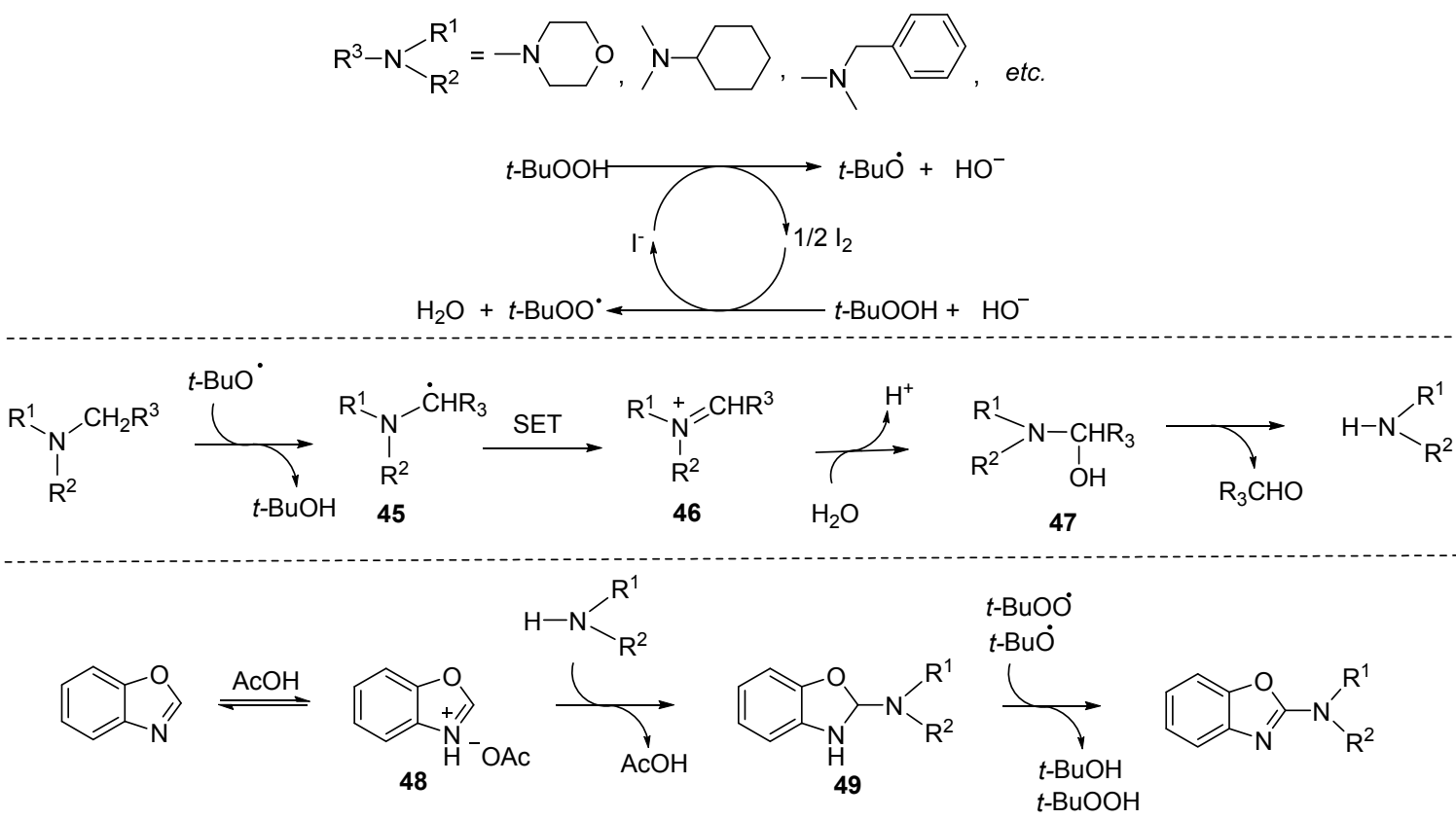

图式 22 合成 2-胺基苯并噁唑衍生物的反应机理

Scheme 22 Reaction mechanism of synthesis of 2-aminobenzoxazole derivatives

3.4 以甲酰胺为胺源合成 2-胺基苯并噁唑衍生物

\subsection{1 过渡金属催化下苯并噁唑 $\mathrm{C}$ (2)直接胺基化}

2009 年, Chang 等 ${ }^{[66]}$ 以取代苯并啞唑与甲酰胺为原

料, $\mathrm{Ag}_{2} \mathrm{CO}_{3}$ 为催化剂, 对甲氧基苯甲酸为辅助剂, 在

$130{ }^{\circ} \mathrm{C}$ 下反应 $12 \mathrm{~h}$, 得到 16 个 2-胺基苯并啞唑衍生物, 产率为 $41 \% \sim 91 \%$ (Eq. 28). 利用该反应条件, 以取代
苯并啞唑与二级胺为原料, 成功合成了目标产物, 产率 为 $49 \% \sim 86 \%$. 当具有一定光学活性的甲酰胺为原料时, 利用该合成方法得到的 2-胺基苯并噁唑衍生物光学活 性没有变化，没有发生外消旋化(Eq. 29).

2011 年, $\mathrm{Li}$ 等 ${ }^{[42]}$ 以取代苯并噁唑与甲酰胺为原料, $\mathrm{Cu}(\mathrm{OAc})_{2} \cdot \mathrm{H}_{2} \mathrm{O}$ 为催化剂, $\mathrm{PhCOOH}$ 为辅助剂, 在氧气的 


$$
\text { 散 }
$$$$
\mathrm{R}=\mathrm{CH}_{3}, \mathrm{OCH}_{3}, \mathrm{Cl}, \mathrm{NO}_{2}, \mathrm{Ph} \text {, etc. }
$$<smiles>[R][R]N([R])c1nc2cc[R]([H])cc2o1</smiles>

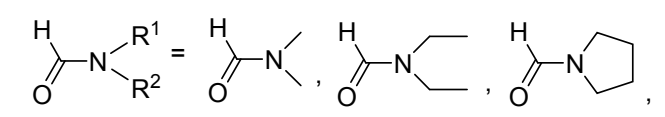<smiles>O=CC1CCCCC1NC(=O)N1CCCCC1</smiles>

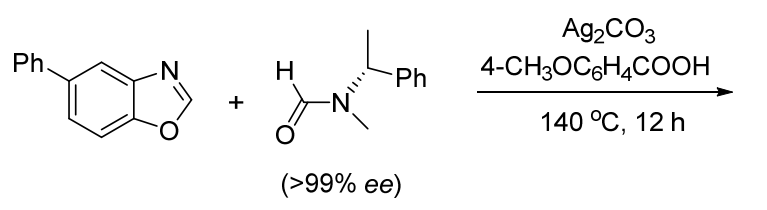<smiles>CC(c1ccccc1)N(C)c1nc2ccccc2o1</smiles>

( $70 \%$ yield, $>99 \%$ ee $)$

氛围中 $130{ }^{\circ} \mathrm{C}$ 条件下反应 $12 \mathrm{~h}$, 合成了 8 个 2-胺基苯并 噁唑衍生物, 产率为 $29 \% \sim 70 \%$ (Eq. 30).

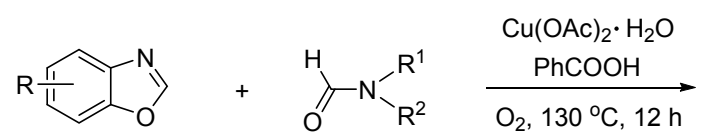

$\mathrm{R}=\mathrm{CH}_{3}, \mathrm{Cl}, \mathrm{t}-\mathrm{Bu}$, etc.

$\mathrm{R}=\mathrm{CH}_{3}, \mathrm{C}_{2} \mathrm{H}_{5}$, etc.

$$
\overbrace{0}^{N} N_{\mathrm{R}^{2}}^{-R^{1}}
$$

2011 年, $\mathrm{Yu}$ 等 ${ }^{[67]}$ 以取代苯并噁唑与甲酰胺为原料, $\mathrm{FeCl}_{3}$ 为催化剂, 咪唑为辅助剂, 在 $130{ }^{\circ} \mathrm{C}$ 下反应 $12 \mathrm{~h}$, 得到 20 个 2-胺基苯并噁唑衍生物, 产率为 12\% 95\% (Eq. 31). 该反应的机理可能是: 苯并噁唑与 $\mathrm{FeCl}_{3}$ 反应 形成盐 50; 甲酰胺在催化剂作用下形成二级胺; 然后 50 与二级胺发生亲核加成反应得到中间体 51 ; 最后 51 在 $\mathrm{Fe}$ (III)作用下脱去 $\mathrm{H}$ 生成目标产物(Scheme 23). 该方法 对于合成 1,3,4-啞二唑的胺基化产物也有较好的效果, 同时, 利用其它的酰胺、二级胺为胺源, 在该条件下, 也 能获得 2-胺基苯并啞唑衍生物, 且具有适中的产率.

\subsection{2 非金属催化下苯并噁唑 $\mathrm{C}(2)$ 直接胺基化}

2014 年, Wang 等 ${ }^{[68]}$ 以甲酰胺类化合物为胺源, 对 取代的苯并噁唑的 2-位直接进行了胺基化, 该方法使用 $20 \mathrm{~mol} \%$ 四丁基碘化铵(TBAI)为催化剂, TBHP 为氧化 剂, $\mathrm{AcOH}$ 为辅助剂, 在 DCE 溶剂中 $90{ }^{\circ} \mathrm{C}$ 下反应 $12 \mathrm{~h}$,
R $\mathrm{R}=\mathrm{CH}_{3}, \mathrm{OCH}_{3}, \mathrm{Cl}, \mathrm{Br}, \mathrm{NO}_{2}, \mathrm{Ph}$, etc.<smiles></smiles><smiles>O=CNC1CCCCC1</smiles><smiles>[R]N([R])c1nc2ccccc2o1</smiles>

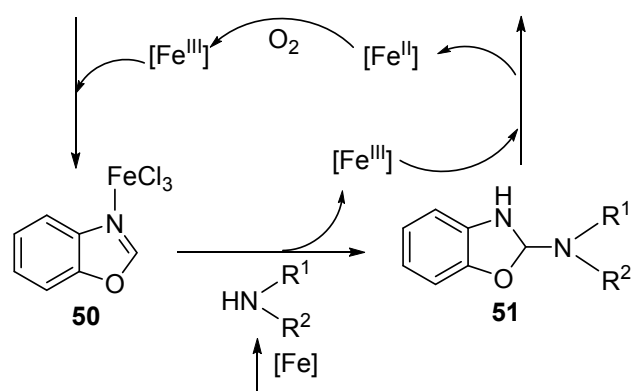

$$
\prod_{\mathrm{O}}^{\mathrm{H}}-\mathrm{N}_{\mathrm{R}^{2}}-\mathrm{R}^{1}
$$

图式 23 合成 2-胺基苯并噁唑衍生物的反应机理 Scheme 23 Reaction mechanism of synthesisi of compound 7 成功地合成了 21 个取代的 2-胺基苯并噁唑衍生物, 产 率达 28\% 87\% (Eq. 32). 该反应可能的机理是: 苯并 啞唑与 $\mathrm{AcOH}$ 反应生成盐 52 ; 甲酰胺类化合物在叔丁氧 基和叔丁过氧基作用下生成胺基自由基 53 ; 苯并噁唑 在叔丁氧基和叔丁过氧基作用下生成苯并噁唑碳自由 基 54; 然后 53 和 54 结合生成目标产物; 52 和 53 结合生 成中间体 55, 55 在 TBHP 作用下, 然后再脱去一个质子 生成目标产物(Scheme 24).

\section{5 以其它含 $\mathrm{N}$ 化合物为胺源合成 2-胺基苯并噁唑衍 生物}

2010 年, Masahiro 等 ${ }^{[69]}$ 利用乙酰丙酮铜/2,2'-吡啶 $\left[\mathrm{Cu}(\mathrm{caca})_{2} / \mathrm{bpy}\right]$ 为催化剂, $N, N-$ 二烷基氯胺类化合物为胺 源, 对苯并噁唑直接进行胺化反应得到 2-氨基苯并噁唑 衍生物. 反应体系以甲苯为溶剂, 叔丁氧基锂 $(\mathrm{LiO} t-\mathrm{Bu})$ 用作碱, 在氮气保护条件下, 室温反应 $2 \mathrm{~h}$, 得到一系列 目标产物，产率为 $17 \% \sim 76 \%$ (Eq. 33).

2012 年 Chen 等 ${ }^{[70]}$ 以取代的苯并噁唑与胺基磺酰氯 为原料, $\mathrm{BipyPdCl} \mathrm{P}_{2}$ 和 $\mathrm{CuCN}$ 为催化剂, $\mathrm{K}_{2} \mathrm{CrO}_{7}$ 为氧化剂, $\mathrm{K}_{2} \mathrm{CO}_{3}$ 用作碱, 氯苯为溶剂, $4 \AA$ 分子篮作用下, 在 


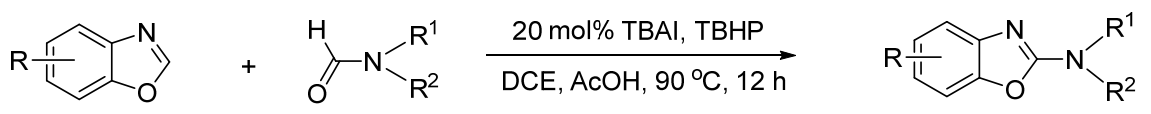

$\mathrm{R}=\mathrm{CH}_{3}, \mathrm{OCH}_{3}, \mathrm{Cl}, \mathrm{Br}, \mathrm{NO}_{2}, t-\mathrm{Bu}, \mathrm{Ph}$, etc. $\quad \mathrm{R}^{1}=\mathrm{R}^{2}=\mathrm{Me}, \mathrm{Et}, n-\mathrm{Pr}, n-\mathrm{Bu}$, etc.

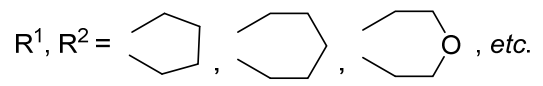

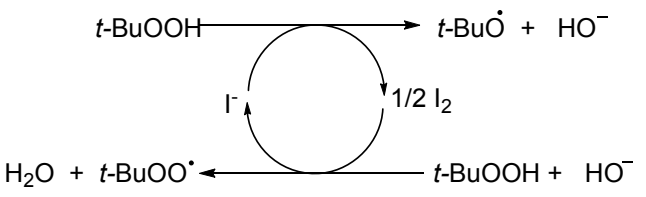

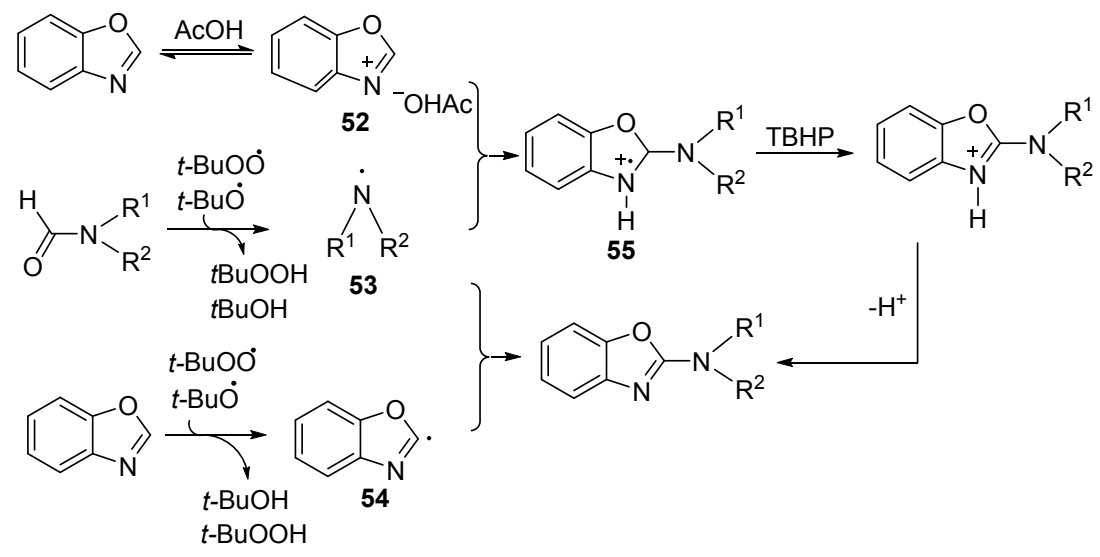

图式 24 合成 2-胺基苯并噁唑衍生物的反应机理

Scheme 24 Reaction mechanism of synthesis of 2-aminobenzoxazole derivatives

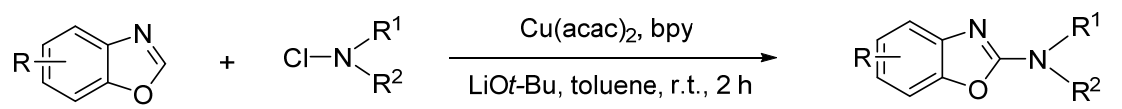

$\mathrm{R}=\mathrm{H}, \mathrm{CH}_{3}, \mathrm{Cl}, \mathrm{Ph}$, etc.<smiles></smiles><smiles>CCCCN(Cl)CCCC</smiles><smiles>CN(Cl)Cc1ccccc1</smiles>

$150{ }^{\circ} \mathrm{C}$ 条件下反应 $24 \mathrm{~h}$, 得到 15 个 2 -胺基苯并亞唑衍 生物, 产率为 $33 \% \sim 85 \%$ (Eq. 34). 该反应可能的机理 是：苯并啞唑首先与 $\mathrm{Cu}(\mathrm{I})$ 反应生成铜盐 56 ; 然后与 $\operatorname{Pd}(\mathrm{II})$ 进行金属交换生成中间体 57; 中间体 57 与胺基磺 酰氯作用生成 $\mathrm{Pd}(\mathrm{IV})$ 的中间体 $\mathbf{5 8}$, 其脱去 $\mathrm{SO}_{2}$, 生成中 间体 59; 59 经过一个还原消除反应生成目标产物 (Scheme 25). 该方法使用到昂贵的金属催化剂, 强的氧 化剂, 且反应温度较高, 时间较长.

2013 年, Yotphan 等 ${ }^{[71]}$ 以 $O$-苯甲酰基差胺为胺源, $\mathrm{CuCl}$ 为催化剂, $\mathrm{PPh}_{3}$ 和 $\mathrm{LiO} t \mathrm{Bu}$ 为碱, $\mathrm{THF}$ 为溶剂, 与取 代的苯并啞唑在室温下反应 $1 \mathrm{~h}$, 合成了 15 个 2-胺基苯 并噁唑衍生物, 产率 45\% 91\% (Eq. 35). 反应的机理 可能是: $\mathrm{CuCl}, \mathrm{PPh}_{3}$ 和 $\mathrm{LiO} t \mathrm{Bu}$ 首先作用生成 $\mathrm{Cu}(\mathrm{I})$ 的复合 物 60, 然后 60 与苯并噁坐反应生成中间体 61; 61 与 $O-$

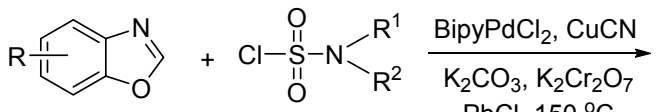

$$
\begin{aligned}
& \mathrm{R}=\mathrm{CH}_{3}, \mathrm{OCH}_{3}, \mathrm{Cl}, \mathrm{NO}_{2}, \mathrm{Ph} \text {, etc. } \\
& \underbrace{\mathrm{N}}_{0} \mathrm{~N}_{\mathrm{R}^{2}}^{-\mathrm{R}^{1}}
\end{aligned}
$$

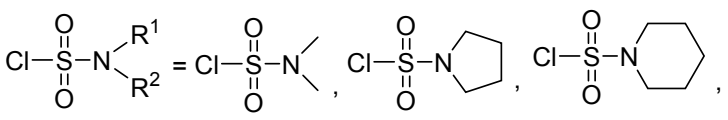

$$
\begin{aligned}
& \text { Cl- }
\end{aligned}
$$

苯甲酰基羟胺反应生成中间体 62,62 通过还原消除生成 


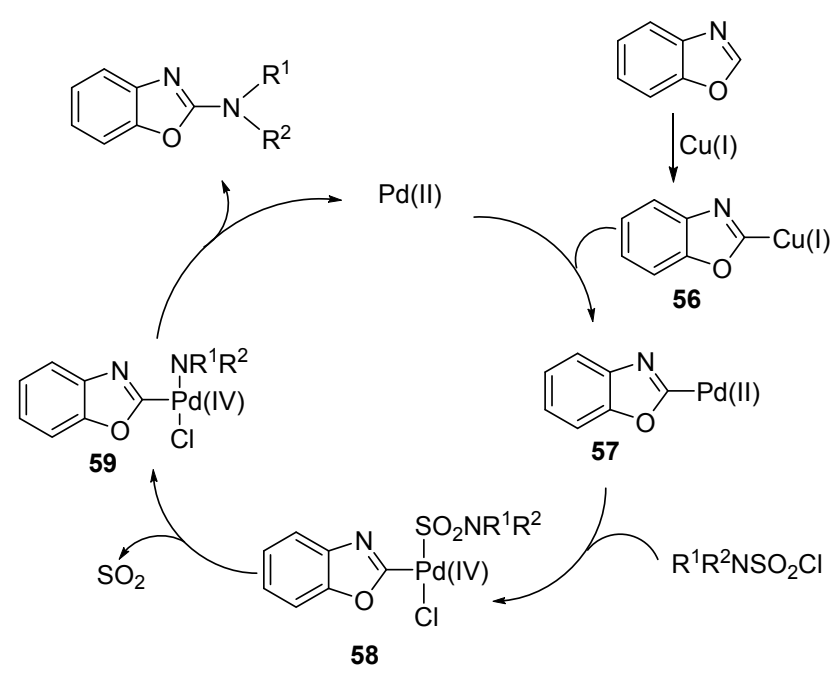

图式 25 合成 2-胺基苯并噁唑衍生物的反应机理

Scheme 25 Reaction mechanism of synthesis of 2-aminobenzoxazole derivatives

2-胺基苯并噁唑衍生物，同时脱去 $\mathrm{Cu}(\mathrm{I})$ 的复合物 63 (Scheme 26).

$$
\begin{aligned}
& \text { ( } \\
& R \frac{1}{4} \underbrace{N}_{0} N_{R^{2}}^{-R^{1}}
\end{aligned}
$$
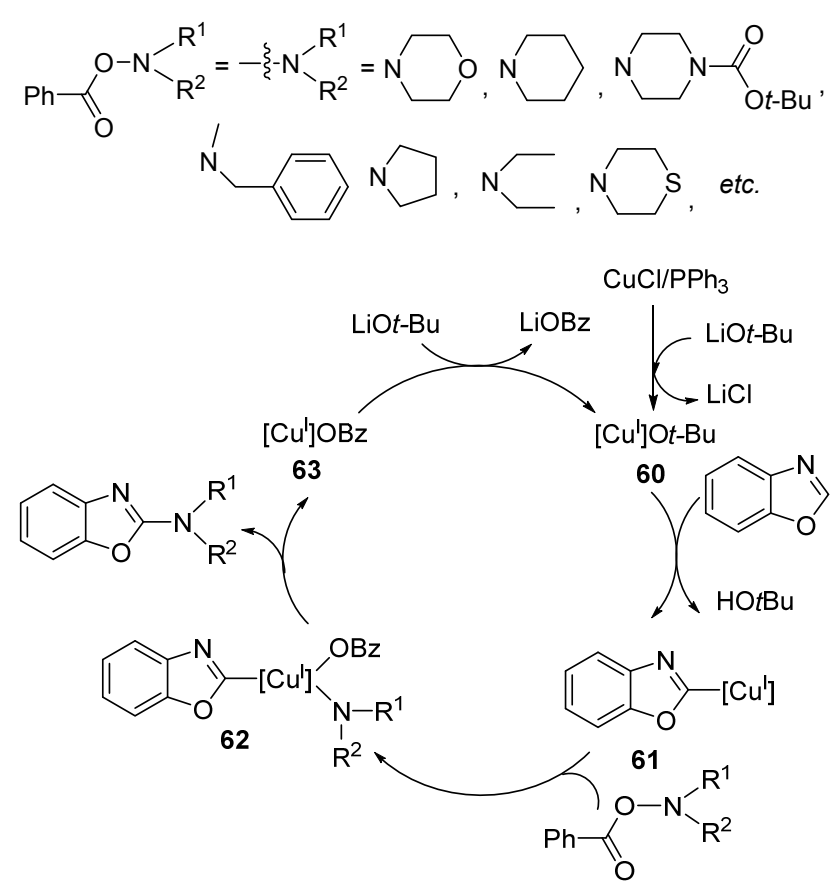

图式 26 合成 2-胺基苯并噁唑衍生物的反应机理 Scheme 26 Reaction mechanism of synthesis of 2-aminobenzoxazole derivatives
2011 年, Hirano 等 ${ }^{[72,73]}$ 以 $O$-苯甲酰基 $N, N$-二乙基羟 胺为胺源, $\mathrm{Cu}(\mathrm{OAc})_{2}$ 为催化剂, $\mathrm{LiO} t$ - $\mathrm{Bu}$ 为碱, 1,4 -二氧六 环为溶剂, 与苯并噁唑在室温下反应 $4 \mathrm{~h}$, 合成了 2-( $N, N$-二乙基)胺基苯并噁唑，产率为 $57 \%$; 同时，该方 法还适用与多氟取代的苯或杂环化合物与 $O$-苯甲酰基 羟胺反应进行直接胺基化反应.

\section{4 以其它原料合成 2-胺基苯并噁唑衍生物}

在有机化学反应过程中, 由于进攻试剂的作用或介 质的影响，发生基团的迁移、碳架的变化乃至环状化合 物环的扩大或缩小、重键位移以致电子云重新排布的重 排反应是很常见的. 利用分子内的重排反应来合成 2-胺 基苯并噁唑衍生物是另一种途径和方法. 例如, 1988 年, Gilchrist 等 ${ }^{[74]}$ 以 1,2,4-苯并啞嗪衍生物为原料, 首先在 $\mathrm{NaOH}$ 溶液中使 3 位酯基发生水解, 然后酸性条件下脱 酸，并通过分子内的重排反应得到中间体 64; 最后再通 过分子内的重排反应生成目标产物(Eq. 36).

$$
\longrightarrow \text { (2) }
$$

2015 年, Yoshida 等 ${ }^{[75]}$ 以 2-嘧啶氧基苯为原料, 在 $\mathrm{LiClO}_{4} / \mathrm{CH}_{3} \mathrm{CN}$ 溶液中, $\mathrm{K}_{2} \mathrm{CO}_{3}$ 用作碱, 通过电解池的电 极氧化的方法, 首先生成一个环状的嘧啶阳离子 $\mathbf{6 5}$; 然 后在哌啶作用下, $70{ }^{\circ} \mathrm{C}$ 下反应 $3 \mathrm{~h}$ 得到目标产物 (Scheme 27). 可能的反应机理是: 2-嘧啶氧基苯在电极 作用条件下失去一个电子生成中间体 $66 ; 66$ 通过分子内 重排关环得到 67;67 失去一个电子和一个质子后生成 68; 在哌啶作用下，反应生成化合物 69; 69 通过重排开 环生成 70; 70 再与一个哌啶反应, 脱去一个哌啶盐后生 成目标产物(Scheme 28).

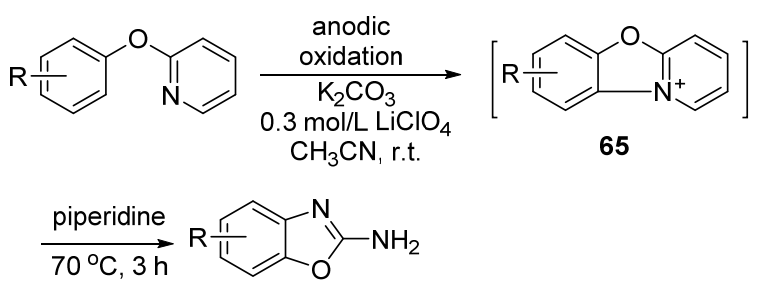

图式 27 合成 2-胺基苯并噁唑衍生物的合成路线

Scheme 27 Synthetic route of 2-aminobenzoxazole derivatives

\section{5 展望}

综上所述，随着 2-胺基苯并噁唑衍生物在医药、农 


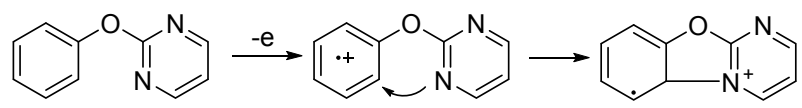

66

67

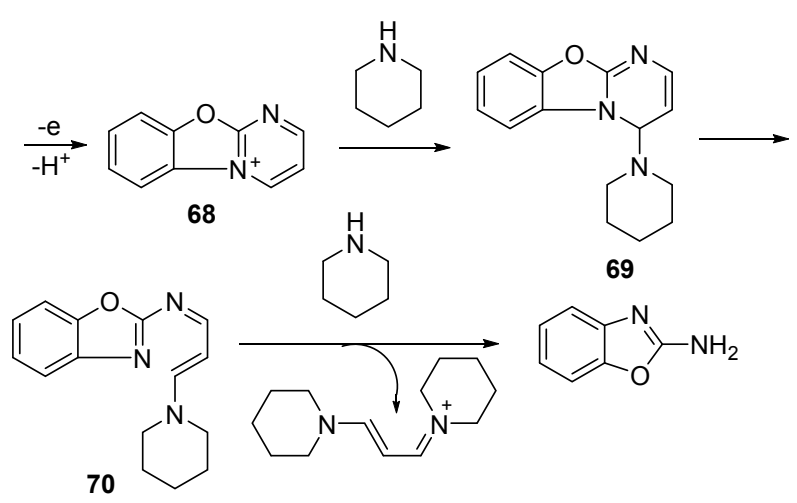

图式 28 合成 2-胺基苯并噁唑衍生物的反应机理

Scheme 28 Reaction mechanism of synthesis of 2-aminobenzoxazole derivatives

药、生物及材料等领域应用范围越来越广泛, 有关其合 成方法的报道也逐年增多. 本文分别以邻氨基苯酚、2取代苯并噁唑、苯并噁唑等为原料, 对 2-胺基苯并噁唑 衍生物的合成方法进行了归纳和总结, 尤其是对苯并啞 唑 2 位 $\mathrm{C}-\mathrm{H}$ 键的直接官能团化合成 2-胺基苯并噁唑衍 生物, 分别以一级胺、二级胺、三级胺、甲酰胺和其它 胺类化合物为胺源进行了详细的综述. 文中所述合成方 法各有其优缺点, 能够适用不同原料和条件下目标产物 的合成.

近年来, 随着研究的深入, 一些高效、环境友好、 绿色环保的合成方法如微波促进、无溶剂或水为溶剂进 行合成、非金属催化剂的使用等逐步被发现, 这是 2-胺 基苯并噁唑衍生物合成方法发展的趋势, 符合绿色化学 的理念. 经过大家的努力, 更多地合成 2-胺基苯并惡唑 衍生物新方法能被发现.

\section{References}

[1] Alper-Hayta, S.; Arisoy, M.; Temiz-Arpaci, Ö.; Yildiz, I.; Aki, E.; Özkan, S.; Kaynak, F. Eur. J. Med. Chem. 2008, 43, 2568.

[2] Safak, C.; Erdogan, H.; Palaska, E.; Sunal, R.; Duru, S. J. Med. Chem. 1992, 35, 1296.

[3] Hal, I. H.; Peaty, N. J.; Henry, J. R.; Easmon, J.; Heinisch, G.; Pürstinger, G. Arch. Pharm. 1999, 332, 115.

[4] Ge, F.; Wang, Z.; Wan, W.; Lu, W.; Hao, J. Tetrahedron Lett. 2007, $48,3251$.

[5] Ren, P.; Salihu, I.; Scopelliti, R.; Hu, X. L. Org. Lett. 2012, 14, 1748.

[6] Wu, X. J.; See, J. W. T.; Xu, K.; Hirao, H.; Roger, J.; Hierso, J. C.; Zhou, J. R. Angew. Chem., Int. Ed. 2014, 53, 13573.

[7] Yang, K.; Chen, X. Y.; Wang, Y. Q.; Li, W. Q.; Kadi, A. A.; Fun, H. K.; Sun, H.; Zhang, Y.; Li, G. G.; Lu, H. J. J. Org. Chem. 2015, 80, 11065.

[8] Yang, K.; Zhang, C.; Wang, P.; Zhang, Y.; Ge, H. B. Chem. Eur. J.
2014, 20, 7241.

[9] Wu, X. F.; Anbarasan, P.; Neumann, H.; Beller, M. Angew. Chem., Int. Ed. 2010, 49, 7316.

[10] Huang, J. K.; Chan, J.; Chen, Y.; Borths, C. J.; Baucom, K. D.; Larsen, R. D. Faul, M. M. J. Am. Chem. Soc. 2010, 132, 3674.

[11] Zhu, F.; Wang, Z. X. Org. Lett. 2015, 17, 1601.

[12] Shibahara, F.; Yamaguchi, E.; Murai, T. Chem. Commun. 2010, 46, 2471.

[13] Matsuyama, N.; Hirano, K.; Satoh, T.; Miura, M. Org. Lett. 2009, $11,4156$.

[14] Kim, S. H.; Yoon, J.; Chang, S. Org. Lett. 2011, 13, 1474.

[15] Parsharamulu, T.; Reddy, P. V.; Likhar, P. R.; Lakshmi, M. Tetrahedron 2015, 71, 1975.

[16] Hou, C. D.; Ren, Y. L.; Lang, R.; Hu, X. X.; Xia, C. G.; Li, F. W. Chem. Commun. 2012, 48, 5181.

[17] Kim, J. Y.; Cho, S. H.; Joseph, J.; Chang, S. Angew. Chem., Int. Ed. 2010, 49, 9899.

[18] Neyts, J.; De Clercq, E.; Singha, R.; Chang, Y. H.; Das, A. R.; Chakraborty, S. K.; Hong, S. C.; Tsay, S. C.; Hsu, M. H.; Hwu, J. R. J. Med. Chem. 2009, 52, 1486.

[19] Cox, C. D.; Breslin, M. J.; Whitman, D. B.; Schreier, J. D.; McGaughey, G. B.; Bogusky, M. J.; Roecker, A. J.; Mercer, S. P.; Bednar, R. A.; Lemaire, W.; Bruno, J. G.; Reiss, D. R.; Harrell, M.; Murphy, K. L.; Garson, S. L.; Doran, S. M.; Prueksaritanont, T.; Anderson, W. B.; Tang, C.; Roller, S.; Cabalu, T. D.; Cui, D. H.; Hartman, G. D.; Young, S. D.; Koblan, K. S.; Winrow, C. J.; Renger, J. J.; Coleman, P. J. J. Med. Chem. 2010, 53, 5320.

[20] Seregin, I.; Gevorgyan, V. Chem. Soc. Rev. 2007, 36, 1173.

[21] Xiao, L. W.; Gao, H. J.; Kong, J.; Liu, G. X.; Peng, X. X.; Wang, S. J. Chin. J. Org. Chem. 2014, 34, 1048.

(肖立伟, 高红杰, 孔洁, 刘光仙, 彭晓霞, 王树军, 有机化学, 2014, 34, 1048.)

[22] Kaupp, G.; Schmeyers, J.; Boy, J. Chem. Eur. J. 1998, 4, 2467.

[23] Wu, Y. Q.; Limburg, D. C.; Wilkinson, D. E.; Hamilton, G. S. J. Heterocycl. Chem. 2003, 40, 191.

[24] El-Faham, A.; Chebbo, M.; Abdul-Ghani, M.; Younes, G. Heterocycl. Chem. 2006, 43, 599.

[25] Cioffi, C. L.; Lansing, J. J.; Yüksel, H. J. Org. Chem. 2010, 75, 7942.

[26] Carpenter, R. D.; Kurth, M. J. Nat. Protoc. 2010, 5, 1731.

[27] Zhang, X. Y.; Jia, X. F.; Wang, J. J.; Fan, X. S. Green Chem. 2011, 13,413 .

[28] Guo, X. J.; Guo, Q.; Zhao, W.; Zhang, X. Y. J. Henan Normal Univ. (Nat. Sci. Ed.) 2013, 41, 85 (in Chinese).

(郭晓杰, 郭强, 赵婉, 张新迎, 河南师范大学 (自然科学版), 2013, 41, 85.)

[29] Guntreddi, T.; Allam, B. K.; Singh, K. N. RSC Adv. 2013, 3, 9875.

[30] Kasthuri, M.; Sharath Babu, H.; Shiva Kumar, C.; Nagendra Kumar, P. V. Synlett 2015, 26, 897.

[31] Lin, C. C.; Hsieh, T. H.; Liao, P. Y.; Liao, Z. Y.; Chang, C. W.; Shih, Y. C.; Yeh, W. H.; Chien, T. C. Org. Lett. 2014, 16, 892.

[32] Naga Raju, G. N.; Prasanna, T. S. K. T. World J. Pharm. Sci. 2015, 4, 1082.

[33] Vardhan Reddy, K. H.; Anil Kumar, B. S. P.; Prakash Reddy, V.; Uday Kumar, R.; Nageswar, Y. V. D. RSC Adv. 2014, 4, 45579.

[34] Yamato, M.; Takeuchi, Y.; Hattori, K.; Hashigaki, K. Chem. Pharm. Bull. 1984, 32, 3053.

[35] Saladino, R.; Crestini, C.; Occhionero, F.; Nicoletti, R. Synth. Commun. 1996, 26, 3241.

[36] Kövér, J.; Tímár, T.; Tompa, J. Synthesis 1994, 1124.

[37] Kim, J. Y.; Cho, S. H.; Joseph, J.; Chang, S. Angew. Chem. 2010, $122,10095$. 
[38] Gu, J.; Cai, C. Synlett 2015, 26, 639.

[39] Kim, J. Y.; Cho, S. H.; Joseph, J.; Chang, S. Angew. Chem., Int. Ed. 2010, 49, 9899.

[40] Li, Y. M.; Liu, J.; Xie, Y. S.; Zhang, R.; Jin, K.; Wang, X. N.; Duan, C. Y. Org. Biomol. Chem. 2012, 10, 3715.

[41] Cai, S. F.; He, W.; Li, Y. D.; Yu, X. F.; Zhang, Q.; Li, L. S. $C N$ 102766108, 2012 [Chem. Abstr. 2012, 157, 734720.]

[42] Li, Y. M.; Xie, Y. S.; Zhang, R.; Jin, K.; Wang, X. N.; Duan, C. Y. J. Org. Chem. 2011, 76, 5444.

[43] Monguchi, D.; Fujiwara, T.; Furukawa, H.; Mori, A. Org. Lett. 2009, 11, 1607.

[44] Mitsuda, S.; Fujiwara, T.; Kimigafukuro, K.; Monguchi, D.; Mori, A. Tetrahedron 2012, 68, 3585.

[45] Pal, P.; Giri, A. K.; Singh, H.; Ghosh, S. C.; Panda, A. B. Chem. Asian J. 2014, 9, 2392.

[46] Xie, Y. J.; Qian, B.; Xie, P.; Huang, H. M. Adv. Synth. Catal. 2013, $355,1315$.

[47] Cao, K.; Wang, J. L.; Wang, L. H.; Li, Y. Y.; Yu, X. H.; Huang, Y. W.; Yang, J. X.; Chang, G. J. Synth. Commun. 2014, 44, 2848.

[48] Lamani, M.; Prabhu, K. R. J. Org. Chem. 2011, 76, 7938.

[49] Froehr, T.; Sindlinger, C. P.; Kloeckner, U.; Finkbeiner, P.; Nachtsheim, B. J. Org. Lett. 2011, 13, 3754.

[50] Wagh, Y. S.; Sawant, D. N.; Bhanage, B. M. Tetrahedron Lett. 2012, 53, 3482.

[51] Gao, W. J.; Li, W. C.; Zeng, C. C.; Tian, H. Y.; Hu, L. M.; Little, R. D. J. Org. Chem. 2014, 79, 9613.

[52] Joseph, J.; Kim, J. Y.; Chang, S. Chem. Eur. J. 2011, 17, 8294.

[53] Wertz, S.; Kodama, S.; Studer, A. Angew. Chem., Int. Ed. 2011, 50, 11511.

[54] Wagh, Y. S.; Tiwari, N. J.; Bhanage, B. M. Tetrahedron Lett. 2013, $54,1290$.

[55] Yotphan, S.; Beukeaw, D.; Reutrakul, V. Synthesis 2013, 45, 0936.

[56] Xu, D. Q.; Wang, W. F.; Miao, C. X.; Zhang, Q. H.; Xia, C. G.; Sun, W. Green Chem. 2013, 15, 2975.

[57] Wang, X. E.; Xu, D. Q.; Miao, C. X.; Zhang, Q. H.; Sun, W. Org.
Biomol. Chem. 2014, 12, 3108.

[58] Maity, S.; Zhu, M.; Shinabery, R. S.; Zheng, N. Angew. Chem., Int. Ed. 2012, 51, 222.

[59] Maity, S.; Zheng, N. Angew. Chem., Int. Ed. 2012, 51, 9562.

[60] Allen, L. J.; Cabrera, P. J.; Lee, M.; Sanford, M. S. J. Am. Chem. Soc. 2014, 136, 5607.

[61] Rueping, M.; Zhu, S.; Koenigs, R. M. Chem. Commun. 2011, 47, 8679.

[62] Wang, J. D.; Liu, Y. X.; Xue, D.; Wang, C.; Xiao, J. L. Synlett 2014, $25,2013$.

[63] Guo, S. M.; Qian, B.; Xie, Y. J.; Xia, C. G.; Huang, H. M. Org. Lett. 2011, 13, 522.

[64] Yuan, J. W.; Jin, M.; Yin, Q. Y.; Mao, P.; Qu, L. B. Z. Naturforsch. 2016, $71 b, 317$.

[65] Wang, S.; Wang, J.; Guo, R.; Wang, G.; Chen, S. Y.; Yu, X. Q. Tetrahedron Lett. 2013, 54, 6233.

[66] Cho, S. H.; Kim, J. Y.; Lee, S. Y.; Chang, S. Angew. Chem., Int. Ed. $\mathbf{2 0 0 9}, 48,9127$.

[67] Wang, J.; Hou, J. T.; Wen, J.; Zhang, J.; Yu, J. Q. Chem. Commun. 2011, 47, 3652.

[68] Wang, R.; Liu, H.; Yue, L.; Zhang, X. K.; Tan, Q. Y.; Pan, R. L. Tetrahedron Lett. 2014, 55, 2233.

[69] Kawano, T.; Hirano, K.; Satoh, T.; Miura, M. J. Am. Chem. Soc. 2010, 132, 6900

[70] Chen, S. Y.; Zheng, K.; Chen, F. Tetrahedron Lett. 2012, 53, 6297.

[71] Yotphan, S.; Beukeaw, D.; Reutrakul, V. Tetrahedron 2013, 69, 6627.

[72] Matsuda, N.; Hirano, K.; Satoh, T.; Miura, M. Org. Lett. 2011, 13, 2860.

[73] Matsuda, N.; Hirano, K.; Satoh, T.; Miura, M. Synthesis 2012, 44, 1792.

[74] Gilchrist, T. L.; John Harris, C.; King, F. D.; Peek, M. E. J. Chem. Soc., Perkin Trans. 1 1988, 2169.

[75] Morofuji, T.; Shimizu, A.; Yoshida, J. Chem. Eur. J. 2015, 21, 3211. 